\title{
Comparison of nonlinear solution techniques named arc-length for the geometrically nonlinear analysis of structural systems
}

\author{
TALASLIOĞLU Tuğrul \\ Department of Civil Engineering, Osmaniye Korkut Ata Unv., Karacaoglan Campus, Fakiusagi, Osmaniye, TURKEY \\ ttalaslioglu@osmaniye.edu.tr; talaslioglu@cu.edu.tr
}

Submitted: 21/01/2019

Revised: $01 / 04 / 2021$

Accepted: 12/04/2021

\begin{abstract}
The nonlinearity issue is one of the promising fields in the engineering area. Particularly, the geometric nonlinearity bears big importance for the structural systems showing a tendency of larger deflection. In order to obtain a correct load-deflection relation for the structural system subjected to any external load, an advanced incremental-iterative based approach has to be utilized in the analysis of nonlinear responses. Arc length method has been proven to be the most perfect one among the nonlinear analysis approaches. Thus, it is extensively applied to the structural systems with pin-connected joints. This study attempts to compare two variations of arc length method named "spherical" and "linearized" for the nonlinear analysis of structural system with rigid-connected joints. Also, two different element formulations are utilized to discretize the structural systems. Two open-source coded programs, Opensees and FEAP, are employed for six benchmark structural systems in order to compare the performance of employed arc-length techniques. Furthermore, in order to make a further observation in the nonlinear behavior of application examples, their simulations are not only sketched using graphs, but also displayed through the movies for each of benchmark tests. Consequently, the linearized type arc length technique implemented in FEAP shows a more success with a better prediction of load-deflection relation, noting that Opensees has a big advantage of having an element, which is capable of simulating the geometric nonlinearity.
\end{abstract}

Keywords: Spherical arc length; Linearized arc length; Snap-back; Snap-through; Frame element.

\section{INTRODUCTION}

Both a higher expectation for the structural system with lower cost and a variety in their building material may cause the structural system to be built with a higher slender. Thus, an inclusion of slender member into structural system causes the structural stability problem to emerge. Furthermore, an unexpected failure in the structural system is generally arisen from an incorrect modelling of structural elements. In fact, the stability issue in the structural mechanic has been already handled in association with the concepts of geometric nonlinearity.

The compatibility and equilibrium equations along with the static or kinematic-related boundary conditions and constitutive equations manage the behavior of geometric nonlinearity. A unique differential equation has the ability of comprising one or more of these governing equations, along with the boundary and initial conditions for the structural mechanic problem. However, solely using such a strong form of governing equations is unfortunately not sufficient for solving any of the structural mechanic problems, due to an interaction among the governing equations. Therefore, a variational (weak) form is generally utilized in the structural mechanic problem with various boundary conditions, since it is possible to represent the governing equations by only a functional, which is responsible for approximating the governing equations, thereby using a Euler-Lagrange formulations. Thus, the requirement of satisfying all of 
the boundary conditions is ignored. In the structural mechanic problem, the utilized functional generally indicates the capacity of stored energy in the structural system. In this regard, the structural mechanic problems with small deformations are represented with the functional with either displacement or stress fields (Santos, 2011).

The stability analysis requires a correct identification of critical points (limit, turning, failure, etc.), since the critical points have big importance in the determination of both the stability boundaries and the response form of structural system. In general, the response forms, which are also named equilibrium path, are snap-through, snapback, bifurcation, and their combinations. Particularly, the snap-through buckling has occurred in a way jumping to a new point on the equilibrium path due to a sudden change in the structural system.

The first attempt to identify a critical point for an axially loaded column was made in 1757 by the mathematician Leonhard Euler. Euler directly utilized a strong form in order to derive the classic linear buckling theory. Unfortunately, the classic linear buckling theory is not able to analyze the postbuckling of structural system. Although the various energy-based functional were utilized to predict exact linear buckling load, their computational procedures ignored the prebuckling deformations as in the classic linear buckling theory (Corsanego and Tafanelli, 1980, Masur and Popelar, 1976, Oran, 1967 and Tabarrok and Yuexi, 1989). Thus, the buckling analysis resulted in a prediction of higher linear buckling load for the complex structural system.

The identification of critical points requires a postbuckling analysis. Particularly, the postbuckling analysis is difficult for the nonconservative structural systems, since the governing equations of structural system have to be in an incremental-iterative form. Otherwise, it is impossible to trace the equilibrium path and identify the critical points.

The arc-length method is proven to be a perfect predictor for the nonconservative structural systems (Riks, 1979 and Crisfield, 1981). Thus, it has been improved in a way of various implementations (Crisfield, 1981, Ramm, 1981, Schweizerhof and Wriggers 1986 and Fried, 1984). Crisfield' technique is based on finding the complex roots. Then, Forde and Stiemer (1987) improved Crisfield' technique using an orthogonality principle against the computation of complex roots. Particularly, Crisfield (1997a; 1997b) highlights the computational procedures of arc-length and its variants through several Fortran codes. Teng and Luo (1998) proposed a new arc-length technique, named accumulated arc length. The other arc-length methods are exhaustively overviewed in references Xiao-Zu and Bashir-Ahmed (2004) and Ritto-Correa and Camotim (2008). It is also proved that the arc-length method is an intelligent nonlinear solving tool for different engineering fields (Yuan and Kardomateas, 2018, Habibi and Bidmeshki, 2018, Uddin et al., 2018), (Shokrieh et al. 2017, Nistor et al., 2017, Ray 2016). Particularly, the snap-through problem is solely examined using arc-length approach (Chandra et al., 2012 and 2015, Moghaddasie and Stanciulescu 2013).

In this study, the arc-length method based an incremental-iterative based computational procedure is utilized for the nonlinear analysis of structural system. Firstly, the load is proportionally introduced into structural system. Then, the responses computed depending on a rigidity matrix " $K$ " or flexibility matrix " $F=K^{-1}$ " along with the residual or end forces are utilized for the next iterations. The rigidity and flexibility matrices, residuals, or end forces are derived depending on the member type utilized as simulation model, for example, bar, beam, plate, and shell. In this study, the frame-type elements widely utilized for the rigid-connected joints are used in the simulation of beam-type structural system. However, FEAP and Opensees programs have two different frame element models, in other words, two different beam theories. Thus, the stability problem for the various structural systems is investigated considering two different element formulations, which are generated using the functional with single and multifields along with two different nonlinear analysis approaches. Due to the assumptions in the formulations of employed elements, the results obtained from the nonlinear structural analysis completely vary depending on the sensitivity to the governing parameters of element formulations, for example, the number of elements and integration points. In this regard, two type arc-length methods named "spherical" and "linearized" are utilized to solve the equations of structural systems due to their ability of analyzing the postbuckling for the conservative and nonconservative structural systems. Then, their computing performances are compared considering the number of elements and integration points.

The outline of this study begins with an introduction. The element formulations are considered in the second section. The employed nonlinear structural analysis approaches are presented in section three. Section four is reserved 
for the application of employed nonlinear structural analysis approaches to the benchmark problems and the discussion of results. The conclusion is presented in the last section.

\section{A CONSIDERATION OF BEAM THEORIES FOR FRAME ELEMENT MODELS UTILIZED IN SIMULATIONS}

This section has two subsections. Thus, firstly, the basic concepts for employed beam theories are introduced. Then, the fundamentals for each of the beam theories are separately presented in two subsections. The formulations of stiffness or flexibility matrices including the residual-related computation are given, thereby associating them with the subroutines and objects of FEAP and Opensees programs, respectively.

In the section of introduction, it is mentioned that the best alternative solution of differential equation in the strong form is to use the weak (variational) form, which is represented by a functional. This functional is obtained in a way of using the energy-related principles. In general, the complementary-energy principles are preferably utilized in the structural mechanic (Veubeke, 1965). The principle of minimum total potential energy and alternatively the principle of virtual work are established on the displacement field, which is assumed as the primary unknowns. These principles assume that the transmission of stress is exact, although there exists a possibility of stress discontinuities at the element boundaries. However, the principle of virtual work is preferably utilized in the nonlinear structural models. In fact, the main reason behind the widely usage of the principle of virtual work is that the form of functional is globally convex if the structural system is conservative.

It is also possible that the functional is represented by the principle of maximum total potential energy, which is obtained, thereby introducing a Legendre transform to the principle of minimum total potential energy.

The structural mechanic problems with relatively larger deformations require a consideration of multifields in order to obtain an accurate nonlinear model for the structural systems (Hermann, 1965). Fortunately, the principle of stationary total complementary energy is proven to be an effective functional for simultaneously using displacement and stress fields (Hellinger, 1914). The functional with both single and multifields is widely utilized in the finite element models.

In general, a functional that indicates the stored energy capacity of structural system is utilized to solve the structural mechanic problem.

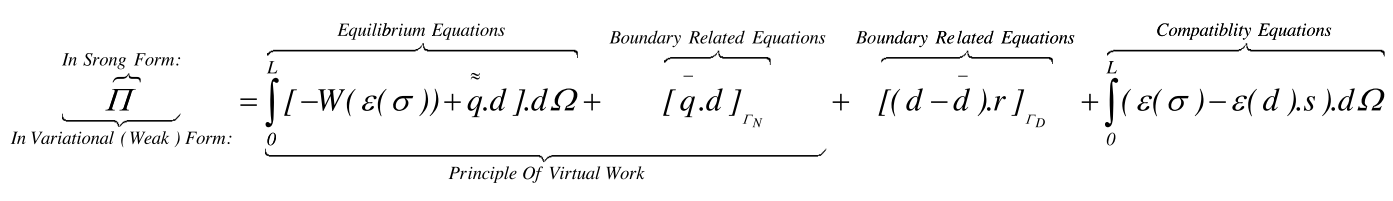

The cross-sections that are divided into small segments throughout the centroidal axis of structural element are laid down in a space $\Omega$. The boundary of space is represented by the combination of Neumann and Dirichlet boundary $\Gamma=\Gamma_{N} \cup \Gamma_{D}$. The generalized vectors $\tilde{q}$ and $\bar{q}$ contain the external distributed and concentrated loads, respectively. While the stress resultant $\sigma$ contains the axial-shear and bending stresses, the generalized strain vector, $\varepsilon$, is composed two vectors, each of which is obtained through the constative equations depending on the axial-shear and bending stresses. Hence, the strain energy density and complementary energy density per unit length of element, $W(\varepsilon(\sigma))$ and $W_{c}(\varepsilon(\sigma))$, respectively, are determined depending on $\varepsilon$. While the generalized vector $\bar{u}$ contains the displacementrotation, the displacement-rotations of element nodes are indicated by the generalized vector $u$. The generalized stress resultants $r$ and reactions $s$ are assumed, defined as $\Omega$ and $\Gamma_{D}$, respectively. In the framework of these definitions, a general expression of potential energy is formulated using the equilibrium, compatibility, and boundary related equations (see Eq. (1))

In Eq. (1), although the potential energy is formulated for multifields, $d, \varepsilon, s$ and $r$, it is easily transformed into the potential energy with single field in a way of omitting several terms. Thus, the principle of virtual work is obtained, 
thereby applying first-order derivation into the potential energy form, which is obtained by discarding several terms. In the structural mechanic, an equilibrium in a structural element is provided only if the variational form of potential energy takes a stationary value for the proposed fields and boundary conditions. In this regard, the variational form of potential energy is firstly equalized to zero. Then, the equilibrium equations are obtained from the Euler-Lagrange equations. The numerical solutions obtained using equilibrium equations and static (Neumann) boundary conditions are named kinematically admissible solutions. Furthermore, it is also mentioned that the displacement field is not commutative due to the larger rotations. Therefore, the rotations are represented by the tensors that is parameterized through Euler-Rodriquez formula (see Reference Ritto-Correa and Camotim, 2002).

The generalized stress resultants, $r$ and reactions, $s$ defined in $\Omega$ and on $\Gamma_{D}$ are represented depending on the generalized operator $H$ that is responsible for computing the rotation tensor as

$$
\begin{aligned}
& s=\sigma \\
& r=n . H . \sigma
\end{aligned}
$$

The strain energy density $W(\varepsilon(\sigma))$ can also be represented depending on the complementary energy density as $W_{c}(\sigma)=W(\varepsilon(\sigma))+\sigma \cdot \varepsilon(\sigma)$. Thus, it is possible to obtain two different functionals depending on two different energy densities. Particularly, the functional obtained using the complementary energy density is also named "HellingerReissner Potential energy form". These functionals are formulated as

$$
\begin{aligned}
& \Pi=\int_{L} \underbrace{[-W(\varepsilon(\sigma))+(\varepsilon(\sigma)}_{W_{c}(\sigma)}-\varepsilon(u) \cdot s)+\tilde{q} \cdot u] \cdot d \Omega+[\bar{q} \cdot u]_{\Gamma_{N}}+[(u-\bar{u}) \cdot \underset{n \cdot H \cdot \sigma}{r}]_{\Gamma_{D}} \\
& \left.\left.\Pi=\int_{L} W_{c}(\sigma)-\varepsilon(u) \cdot \sigma\right)+\tilde{q} \cdot u\right] \cdot d \Omega+[\bar{q} \cdot u]_{\Gamma_{N}}+[(u-\bar{u}) \cdot n \cdot H \cdot \sigma]_{\Gamma_{D}}
\end{aligned}
$$

The numerical solutions obtained using the equilibrium and compatibility equations including the kinematic (Dirichlet) boundary conditions are named statically admissible solutions.

\subsection{The Frame Element Model Used by Opensees Program}

The statically admissible solutions are obtained depending on the total potential energy form, which contains the equilibrium and compatibility equations including the static and kinematic boundary conditions (see Eq. (3)) (Neuenhofer and Filippou, 1997 and Spacone et. al., 1996, Scott and Fenves, 2006). It is also mentioned that a special case of the total energy form is associated with the complementary energy density and named as "Hellinger-Reissner Potential energy form" in the preceding section. Thus, the Hellinger-Reissner Potential energy form in Eq. 4 can be represented by two separate subpotential energy forms:

$$
\Pi_{H R}=\int_{L}[\underbrace{W_{c}(\sigma)-\sigma \cdot \varepsilon(u)}_{\Pi_{t u t}}+\underbrace{\tilde{q} \cdot u] \cdot d \Omega++[\bar{q} \cdot u]_{\Gamma_{N}}+[n \cdot H \cdot \sigma \cdot(u-\bar{u})]_{\Gamma_{D}}}_{\Pi_{e x t}}
$$

The displacements $u$ is represented with the Bernoulli-Euler beam theory neglecting the warping effect, but not the twist effect $\psi$ (see Souza, 2000):

$$
u(x)=\left\{\begin{array}{c}
u(x, y, z) \\
v(x, y, z) \\
w(x, y, z)
\end{array}\right\}=\left\{\begin{array}{c}
u(x)+y v^{\prime}(x)-z w^{\prime}(x) \\
v(x)-z \psi(x) \\
w(x)+y \psi(x)
\end{array}\right\}
$$

Then, the displacements $u_{0}$ and strains $\varepsilon$ depending on the generalized section strains $d$ and the coordinates in cross-section $a$ are represented as 


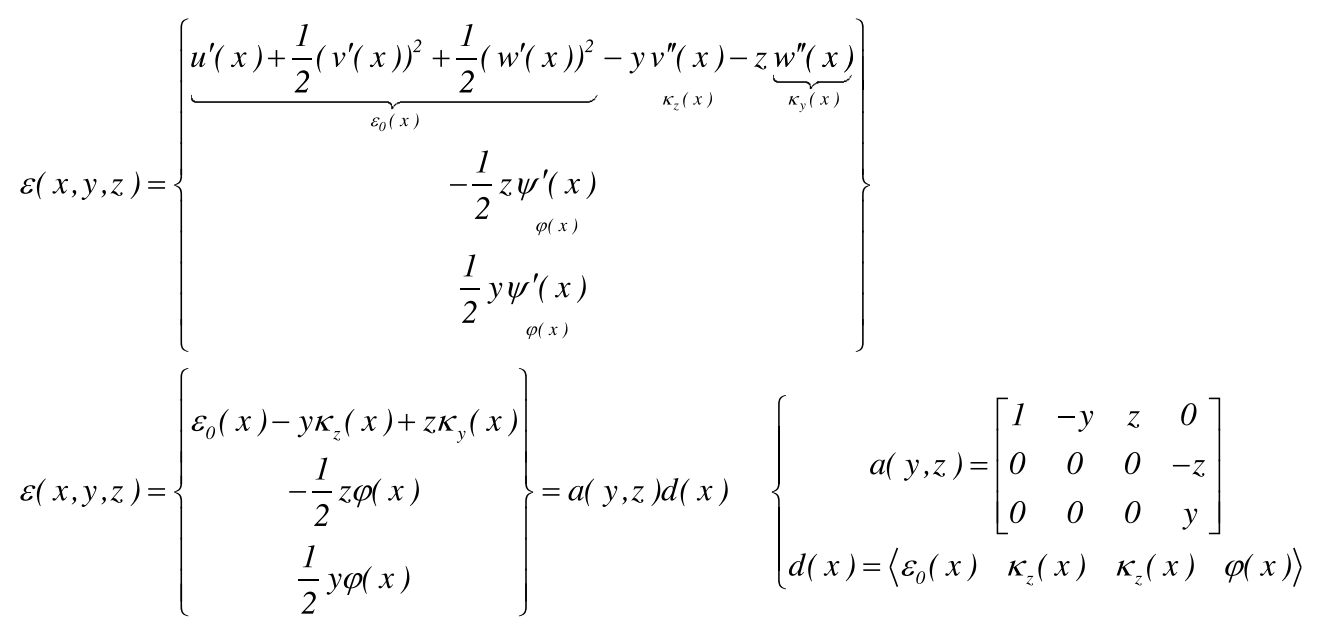

An integration of axial forces $N$, the bending moments $M$ along with the torque $T$ on the cross-sectional area $A$ depending on the stress $\sigma$ give the stress resultant vector $S$ :

$$
\begin{aligned}
& S(x)=\left\langle\begin{array}{llll}
N & M_{z} & M_{y} & T
\end{array}\right\rangle^{T} \\
& S(x)=\left\langle\begin{array}{llll}
\int_{A} \sigma_{x x} d A & -\int_{A} y \sigma_{x x} d A & \int_{A} z \sigma_{x x} d A & \int_{A}\left(\sigma_{x z}-z \sigma_{x y}\right) d A
\end{array}\right\rangle
\end{aligned}
$$

Eq. (8) is also written depending on an assumption of general function $C$ :

$$
S(x)=\int_{A} a(y, z)^{T} \sigma(\varepsilon(x, y, z)) d A=\int_{A} a(y)^{T} \sigma(a(y, z) d(x)) d A=C[d(x)]
$$

In fact, the stress resultant vector in Eq. (9) also represents the section constitutive relation. Therefore, the linearization of this section constitutive relation resulted in the tangent section stiffness matrix $k(d(x))=\frac{\partial C(d(x))}{\partial d(x)}$ or the tangent section flexibility matrix $f(x)=k(x)^{-1}$.

The stationary of the Hellinger-Reissner potential functional in Eq. (5) is computed, thereby taking its variation and setting it equal to zero as

$$
\delta \Pi_{H R}=\frac{\partial \Pi_{H R}}{\partial u_{0}} \delta u_{0}+\frac{\partial \Pi_{H R}}{\partial S} \delta S=0
$$

Furthermore, if the terms $\bar{P}$ and $D$ are assumed to represent the specified end forces and end displacements assuming the moving system in a rigid body modes, the term $\Pi_{e x t}$ becomes equal to $\Pi_{e x t}=\stackrel{-T}{P}^{T} D$. Thus, Eq. (10) is rewritten as

$$
\begin{aligned}
& \frac{\partial \Pi_{H R}}{\partial u_{0}} \delta u_{0}=\int_{L} \int^{T}\left\{\begin{array}{c}
\delta u^{\prime}+v^{\prime} \delta v^{\prime}+w^{\prime} \delta w^{\prime} \\
\delta v^{\prime \prime} \\
-\delta w^{\prime \prime} \\
\delta \psi^{\prime}
\end{array}\right]-\bar{P}^{T} \delta D=0 \\
& \frac{\partial \Pi_{H R}}{\partial S} \delta S=\underbrace{\int_{L} \delta S^{T}\left[\begin{array}{c}
u^{\prime}+\frac{1}{2} v^{\prime 2}+w^{\prime 2} \\
v^{\prime \prime} \\
-w^{\prime \prime} \\
\psi^{\prime}
\end{array}\right]-\underbrace{\frac{\partial W_{c}(\sigma)}{\partial S}}_{(\text {see Eqn [104]) }}] d x}_{\text {Equation(2) }}=0
\end{aligned}
$$


Following the virtual boundary conditions $\delta u(O)=\delta v(O)=\delta v(L)=\delta w(O)=\delta u(L)=\delta \psi(O)=O$ and the basic assumptions $N(L)=P_{1}, M_{z}(O)=-P_{2}, M_{z}(L)=P_{3}, M_{y}(O)=-P_{4}, M_{y}(L)=P_{5}, T(L)=P_{6}$, the solution of "Equation (1)" is in Eq. (11); in other words, the linear and angular momentum balance equations give the section forces $S(x)$, which are represented depending on the end forces $P$ and the displacement-dependent force interpolation functions $b(x)$ :

$$
S(x)=b(x) P\left\{b(x)=\left[\begin{array}{cccccc}
1 & 0 & 0 & 0 & 0 & 0 \\
v\left(\frac{x}{L}\right) & \frac{x}{L}-1 & \frac{x}{L} & 0 & 0 & 0 \\
-w\left(\frac{x}{L}\right) & 0 & 0 & \frac{x}{L}-1 & \frac{x}{L} & 0 \\
0 & 0 & 0 & 0 & 0 & 1
\end{array}\right]\right.
$$

Assuming the virtual forces $\delta N, \delta M_{z}, \delta M_{y}, \delta T$ and the given displacements $u, v$ and $w$, the solution of "Equation (2)" in Eq. (11) gives the virtual section forces $\delta S$, which are represented on the virtual end forces $\delta P$ and the displacement-dependent virtual force interpolation functions $b^{*}(x)$ :

$$
\delta S(x)=b^{*}(x) \delta P\left\{b^{*}(x)=\left[\begin{array}{cccccc}
1 & 0 & 0 & 0 & 0 & 0 \\
\frac{1}{2} v\left(\frac{x}{L}\right) & \frac{x}{L}-1 & \frac{x}{L} & 0 & 0 & 0 \\
-\frac{1}{2} w\left(\frac{x}{L}\right) & 0 & 0 & \frac{x}{L}-1 & \frac{x}{L} & 0 \\
0 & 0 & 0 & 0 & 0 & 1
\end{array}\right]\right.
$$

Furthermore, the integration of virtual section forces $\delta S$ and the generalized section strains $d$ gives the product of virtual end forces $\delta P$ and end displacements $D$ :

$$
\int_{L} \delta S(x)^{T} d(x) d x=\int_{L} b^{*}(x) \delta P d(x) d x=\int_{L} \delta P^{T} \underbrace{b^{*}(x)^{T} d(x) d x}_{D}=\delta P^{T} D
$$

It is noted that Eq. (14) is computed in the section state level. The computation of end displacement $D$ is accomplished using the integration points throughout element length. Furthermore, Eq. (14) has big importance, since it is possible that the flexibility and stiffness matrix is derived for the geometrically nonlinear flexibility-based element in a way of taking the derivative of end displacements $D$ with respect to the end forces $P$ :

$$
F=\frac{\partial D}{\partial P}=\int_{L}\left(b^{*}(x)^{T} f(x)[b(x)+h(x)]+g(x)\right) d \quad \text { or } \quad K=\frac{\partial P}{\partial D}=F^{-1}
$$

where $b(x), b^{*}(x)$ and $f(x)$ are the displacement-dependent force and virtual force interpolation functions (see Eq. (12-13)) and the section tangent flexibility matrix (see Eq. (10)), respectively. Also, $h(x)$ and $g(x)$ are obtained depending on the displacements $u \& w$, the curvatures $\kappa_{z} \& \kappa_{y}$ and the end forces $P_{1}, P_{2}, P_{3}, P_{4}, P_{5}$ and $P_{6}$

$$
h(x)=P_{1}\left[\begin{array}{cccccc}
0 & 0 & 0 & 0 & 0 & 0 \\
\frac{\partial v(x)}{\partial P_{1}} & \frac{\partial v(x)}{\partial P_{2}} & \frac{\partial v(x)}{\partial P_{3}} & \frac{\partial v(x)}{\partial P_{4}} & \frac{\partial v(x)}{\partial P_{5}} & \frac{\partial v(x)}{\partial P_{6}} \\
\frac{\partial w(x)}{\partial P_{1}} & \frac{\partial w(x)}{\partial P_{2}} & \frac{\partial w(x)}{\partial P_{3}} & \frac{\partial w(x)}{\partial P_{4}} & \frac{\partial w(x)}{\partial P_{5}} & \frac{\partial w(x)}{\partial P_{6}} \\
0 & 0 & 0 & 0 & 0 & 0
\end{array}\right]
$$




$$
\begin{aligned}
& g(x)=\frac{1}{2} \kappa_{z}\left[\begin{array}{cccccc}
\frac{\partial v(x)}{\partial P_{1}} & \frac{\partial v(x)}{\partial P_{2}} & \frac{\partial v(x)}{\partial P_{3}} & \frac{\partial v(x)}{\partial P_{4}} & \frac{\partial v(x)}{\partial P_{5}} & \frac{\partial v(x)}{\partial P_{6}} \\
0 & 0 & 0 & 0 & 0 & 0 \\
0 & 0 & 0 & 0 & 0 & 0 \\
0 & 0 & 0 & 0 & 0 & 0 \\
0 & 0 & 0 & 0 & 0 & 0 \\
0 & 0 & 0 & 0 & 0 & 0
\end{array}\right]- \\
& \frac{1}{2} \kappa_{y}\left[\begin{array}{cccccc}
\frac{\partial w(x)}{\partial P_{1}} & \frac{\partial w(x)}{\partial P_{2}} & \frac{\partial w(x)}{\partial P_{3}} & \frac{\partial w(x)}{\partial P_{4}} & \frac{\partial w(x)}{\partial P_{5}} & \frac{\partial w(x)}{\partial P_{6}} \\
0 & 0 & 0 & 0 & 0 & 0 \\
0 & 0 & 0 & 0 & 0 & 0 \\
0 & 0 & 0 & 0 & 0 & 0 \\
0 & 0 & 0 & 0 & 0 & 0 \\
0 & 0 & 0 & 0 & 0 & 0
\end{array}\right]
\end{aligned}
$$

In Eq. (16-17), the terms $\partial \mathrm{v} / \partial \mathrm{P}$ and $\partial \mathrm{w} / \partial \mathrm{P}$ are represented by a matrix notation as

$$
\left\{\begin{array}{l}
\frac{\partial v}{\partial P} \\
\frac{\partial w}{\partial P}
\end{array}\right\}=\left[\begin{array}{cc}
A_{z} & B \\
B & A_{y}
\end{array}\right]^{-1}\left\{\begin{array}{c}
I^{*} a_{z} \\
-I^{*} a_{y}
\end{array}\right\}\left\{\begin{array}{c}
A_{z i j}=\delta_{i j}-I_{i j}^{*} f_{22}\left(\xi_{j}\right) P_{I} \\
A_{y j j}=\delta_{i j}-I_{i j}^{*} f_{33}\left(\xi_{j}\right) P_{l} \\
B_{i j}=I_{i j}^{*} f_{23}\left(\xi_{j}\right) P_{I} \\
a_{z j k}=\sum_{l} f_{2 l}\left(\xi_{j}\right) b_{l k}\left(\xi_{j}\right) \\
a_{y j k}=\sum_{l} f_{3 l}\left(\xi_{j}\right) b_{l k}\left(\xi_{j}\right)
\end{array}\right.
$$

where $I^{*}$ is the curvature-based displacement interpolation influence matrix, and $\xi_{j}$ are sample points that indicate the integration points in the number of $(j=1, . ., n)$ (see Souza, 2000).

It is assumed that the deformations are defined on three coordinate systems, named global that does not vary with the end nodal displacements of structural element, local that indicates a straight line connected the end nodes of deformed structural element, and basic that is obtained from the deformed structural element depending on the rigid body translational and rotational movement. It is noted that the basic system is utilized in the generation of co-rotational type formulation of structural element.

The stiffness matrix $K$ (see Eq. (15) and end forces $P$ are obtained in the basic system. The transformations of these matrices into the local system are provided by the use of a transformation matrix $T$. In fact, the transformation between the basic and local systems is accomplished by using the principle of corotational formulation. The fundamental behind this principle is based on a movement of finite displacement and rigid body rotations, but the moderate or small deformations. Therefore, firstly, the nodal triads $N_{I}=\left[\begin{array}{lll}n_{I 1} & n_{I 2} & n_{I 3}\end{array}\right]$ and $N_{J}=\left[\begin{array}{llll}n_{J 1} & n_{J 2} & n_{J 3}\end{array}\right]$ in the basic system are defined depending on the base vectors $\hat{E}=\left[\begin{array}{lll}\hat{e}_{1} & \hat{e}_{2} & \hat{e}_{3}\end{array}\right]$ in the local system. In fact, the rotation matrix $R$ is represented by the pseudo(rotation) vectors $\gamma_{I}$ and $\gamma_{J}$ that define the rotation of the element ends and are obtained as

$$
R(\gamma)=N_{J} \cdot N_{I}^{T}\left\{\begin{array}{l}
N_{I}=R\left(\gamma_{I}\right) \cdot N_{J}=R\left(\gamma_{I}\right) \hat{E} \\
N_{I}=R\left(\gamma_{J}\right) \cdot N_{J}=R\left(\gamma_{J}\right) \hat{E}
\end{array}\right.
$$


It is noted that the rotation vectors are not additive. Thus, the rotation vector $\gamma$ is moderately large and the mean rotation matrix $\bar{R}=R(\gamma / 2)=\left[\begin{array}{lll}\bar{r}_{1} & \bar{r}_{2} & \bar{r}_{3}\end{array}\right]$ with the unit vectors $\bar{r}$ is a reasonable representation of the rotation from $N_{I}$ to the mean configuration. The computation of $\gamma / 2$ involves the extraction of the unit quaternion from the rotation matrix $R(\gamma)$. In fact, the elements of mean rotation matrix $\bar{R}$ are utilized for the computation of unit vectors $E=\left[\begin{array}{lll}e_{1} & e_{2} & e_{3}\end{array}\right]$ in the basic system. Thus, it is possible to compute the rotations $\theta_{I}$ and $\theta_{J}$ for each of nodes $\mathrm{I}$ and $\mathrm{J}$ in the basic system in a way of using the unit vectors $E$ and the nodal triads $N_{I}$ and $N_{J}$ and thus form the generalized displacement matrix $D$ depending on the rotations $\theta_{I}$ and $\theta_{J}$. Furthermore, it has to be noted that the transformation matrix $T$ is also formed depending on the unit vectors $E$ and the rotations $\theta_{I}$ and $\theta_{J}$.

After the computation of transformation matrix $T$, the element tangent stiffness matrix $\hat{K}$ in the local system is calculated depending on the geometric stiffness matrix $K_{G}$ and element tangent stiffness matrix $K$ in the basic system as

$$
\hat{K}=T^{T} K T+K_{G}
$$

In Eq. (20), the derivation of element geometric stiffness matrix $K_{G}$ is found in Reference Souzo (2000).

\section{Computing Procedure}

1. Update the translational displacement matrix $U$ and the rotational matrix $R$ using $\Delta U$ and $\Delta \gamma$, which is extracted from the general displacement vector $\Delta \hat{D}$ in the local system (see TclForceBeamColumnCommand.cpp and ForceBeam Column3d.cpp):

$$
\begin{aligned}
U_{N N_{\text {Update }}} & =U_{N N_{\text {Prev }}}+\Delta U_{N N} \\
R\left(\gamma_{N N}\right)_{\text {Update }} & =R\left(\Delta \gamma_{N N}\right) R\left(\gamma_{N N}\right)_{\text {Prev }}
\end{aligned}\left\{\Delta \hat{D}_{N N}=\left\{\begin{array}{c}
\Delta U_{N N} \\
\Delta \gamma_{N N}
\end{array}\right\}\right.
$$

Because of the impossibility of summing the rotation vectors $\gamma$, the best way of obtaining the mean rotational matrix $\bar{R}$ is to utilize the quaternions associated with the rotation vectors $\gamma$ (see Eq. (19) (see CorotCrdTransf3d.cpp including subfunctions CorotCrdTransf3d::getQuaternionFromPseudoRotVector, CorotCrdTransf3d::quaternionPro duct, CorotCrdTransf3d::getRotationMatrixFromQuaternion,

CorotCrdTransf3d::getSkewSymMatrix,

CorotCrdTransf3d::getQuaternionFromRotMatrix,

CorotCrdTransf3d::getTangScaledPseudoVectorFromQuaternion, respectively).

2. Compute the unit vectors $E$ in the basic system depending on the mean rotation matrix $\bar{R}$ (see CorotCrdTransf3d::c ompTransfMatrixBasicGlobal)

3. Compute the rotations $\theta_{I}$ and $\theta_{J}$ for each of nodes I and $\mathrm{J}$ in the basic system (see CorotCrdTransf3d::getBasicTri alDisp).

4. Form the generalized displacement matrix $D$ depending on the rotations $\theta_{I}$ and $\theta_{J}$ (see CorotCrdTransf $3 d$.cpp)

5. Compute the displacement $D^{*}$ and $K$ (see the term $D$ in Eq. (14) and $K^{=} F^{-1}$ in Eq. (15)) in the section state level and then utilize $D^{*}$ with the generalized displacement matrix D for the updated displacement matrix $D_{\text {Update }}=D^{*}-D$ (see Souza, 2000). Then, update end forces $P_{\text {Update }}=K . D_{\text {Update }} \mathrm{P}$ in the element state level (see ForceBeamColumn3d.cpp).

6. Compute the transformation matrix $T$ depending on the unit vectors $E$ and the rotations $\theta_{I}$ and $\theta_{J}$ (see $\operatorname{Corot} \operatorname{CrdTr}$ ansf3d::compTransfMatrixBasicGlobal).

7. Compute the geometric stiffness matrix $K_{G}$.

8. Compute the end forces $\hat{P}=T^{T} P_{U p d a t e}$ and tangent stiffness matrices $\hat{K}=T^{T} K T+K_{G}$ in the local system (see ForceBeam Column3d.cpp). 


\subsection{The Frame Element Model Used by FEAP Program}

It is mentioned that the kinematically admissible solutions are obtained through the use of the virtual work principle. In other words, the total potential energy functional, which is constituted on the equilibrium equations and static boundary conditions, is a functional of vectorial field (Carotenuto, 2006, Simo and Vu-Quoc, 1986, Crisfield, 1990, Ibrahimbegovic and Mikdad, 1998, Zienkiewicz and Taylor, 2000a and 2000b).

$$
\Pi=\Pi_{I n t}-\Pi_{E x t}
$$

If its incremental form for the solution with a numerical solution, for example, Newton-Raphson, is considered, then it is written as

$$
\delta \Pi=\Pi_{I n t}-\Pi_{E x t}+\delta \Pi_{I n t}=0
$$

It is assumed that a vector $\hat{P}$ is obtained, thereby rotating the vector $P$ by an angle $\boldsymbol{\theta}$. In fact, the rotation $\boldsymbol{\theta}$ in the vector form contains the angles $\phi, \chi, \psi$, each of which represents the related angle components in the cartesian coordinate system. Thus, the rotation vector $\boldsymbol{\theta}$ is written depending on the unit vector $e$ and its normalized form $\theta$ :

$$
\theta=\theta e\left\{\theta=\sqrt{\left(\phi^{2}+\chi^{2}+\psi^{2}\right)}\right.
$$

Then, the relation between $\hat{P}$ and $\mathrm{P}$ can be associated with a three-dimensional rotation tensor $\Lambda(\boldsymbol{\theta})$ :

$$
\hat{P}=\Lambda(\theta) P
$$

The rotation vector parametrization of rotation tensor $\Lambda(\theta)$ is also accomplished depending on the normalized form $\theta$ :

$$
\Lambda(\boldsymbol{\theta})=I+\frac{\sin \theta}{\theta} \Theta+\frac{1-\cos \theta}{\theta^{2}} \Theta^{2}\left\{\begin{aligned}
\Theta & =\left[\begin{array}{ccc}
0 & -\psi & \chi \\
\psi & 0 & - \\
- & \phi & 0
\end{array}\right] \\
\Theta^{2} & =\left[\begin{array}{ccc}
-\left(\chi^{2}+\psi^{2}\right) & \chi \phi & \phi \psi \\
\chi \phi & -\left(\psi^{2}+\phi^{2}\right) & \psi \chi \\
\phi \psi & \psi \chi & -\left(\phi^{2}+\chi^{2}\right)
\end{array}\right]
\end{aligned}\right.
$$

It is noted that this parametrization is also called as "exponential mapping" or "Rodrigues formulae".

Furthermore, the axial vector $\omega$ is also obtained depending on the derivative of the rotation vector $\dot{\theta}$ :

$$
\omega=T(\theta) \dot{\theta} \quad\left\{T(\theta)=I+\frac{1-\cos \theta}{\theta^{2}} \Theta+\frac{\theta-\sin \theta}{\theta^{3}} \Theta^{2}\right.
$$

It is possible that the linearization of $T(\boldsymbol{\theta})$ and $\Lambda(\boldsymbol{\theta})$ is computed as $\delta T$ and $\delta \Lambda$. Thus, the direction vector $w_{\delta}$ is represented:

$$
w_{\delta}=T(\boldsymbol{\theta}) \delta \boldsymbol{\theta}
$$

The other important issue is related to the alternative representation of rotation vector $\boldsymbol{\theta}$ and its normalized form $\theta$ due to the barriers on the additive feature of vectorial quantities. Thus, the quaternion q, which contains both the scalar part $q_{0}$ and vector part $\boldsymbol{q}$, is utilized to represent the rotation tensor $\Lambda(\boldsymbol{\theta})$ : 


$$
\Lambda(\boldsymbol{\theta})=\left(2 q_{0}^{2}-1\right) \boldsymbol{I}+2 q_{0} \boldsymbol{Q}+2 \boldsymbol{q} \otimes \boldsymbol{q} \ldots\left\{\begin{array}{l}
\left\{q_{0}, \boldsymbol{q}\right\}=\left\{\cos \frac{\theta}{2}, \boldsymbol{\theta} \sin \frac{\theta}{2}\right\} \\
\boldsymbol{Q}=q \times(\text { A skew }- \text { tensor with axial vector } \boldsymbol{q} \\
\boldsymbol{I} \text { (Identy matrix) }
\end{array}\right.
$$

It is assumed that the unity vector $E_{3}$ and $t_{3}$ defines the third axis for the undeformed and deformed configuration of element throughout the element length $L$ along with the unity vectors $E_{1} \& E_{2}$ and $t_{1}$ and $t_{2}$ for the undeformed and deformed configuration of element cross-section, respectively. Then, the position vector $\boldsymbol{X}$ for the undeformed configuration is

$$
\boldsymbol{X}=X_{\alpha} E_{\alpha}+X_{3} E_{3}\left\{\begin{array}{l}
X_{1}, X_{2} \in \text { Cross }- \text { section } \\
X_{3} \in \text { Element Length }
\end{array}\right.
$$

The position vector $\boldsymbol{x}$ for the deformed configuration is computed depending on the deformation map $\phi\left(X_{1}, X_{2}, X_{3}\right)$ and a vector field $\phi_{0}\left(X_{3}\right)$ for the position of line centroid:

$$
\boldsymbol{x}=\phi\left(X_{1}, X_{2}, X_{3}\right)=\phi_{0}\left(X_{3}\right)+X_{\alpha} t_{\alpha}\left(X_{3}\right)=\phi_{0}\left(X_{3}\right)+X_{\alpha} \Lambda\left(X_{3}\right) E_{\alpha}
$$

It is noted that the rotation tensor $\Lambda(\theta)$ is also utilized to represent the cross-section rotation $\Lambda\left(X_{3}\right)$. Thus, a general expression for the rotation tensor $\Lambda$ can be reformulated using the vector product $\otimes$ :

$$
\Lambda=t_{\alpha} \otimes E_{\alpha}
$$

The derivative of cross-section rotation $\Lambda\left(X_{3}\right)$ is

$$
\frac{\partial \Lambda\left(X_{3}\right)}{\partial X_{3}}=\Lambda_{3}
$$

The deformation gradient $\boldsymbol{F}$ from the three-dimensional theory of nonlinear continuum mechanics is

$$
\boldsymbol{F}=\frac{\partial \phi}{\partial \boldsymbol{X}} \text { or } F_{i j}=\frac{\partial x_{i}}{\partial X_{j}}
$$

Thus, the deformation gradient $\boldsymbol{F}$ is written using the strains $\gamma_{r}$ and $\kappa_{r}$ for the undeformed configuration of element along with $\gamma$ and $\kappa$ for the deformed configuration of element:

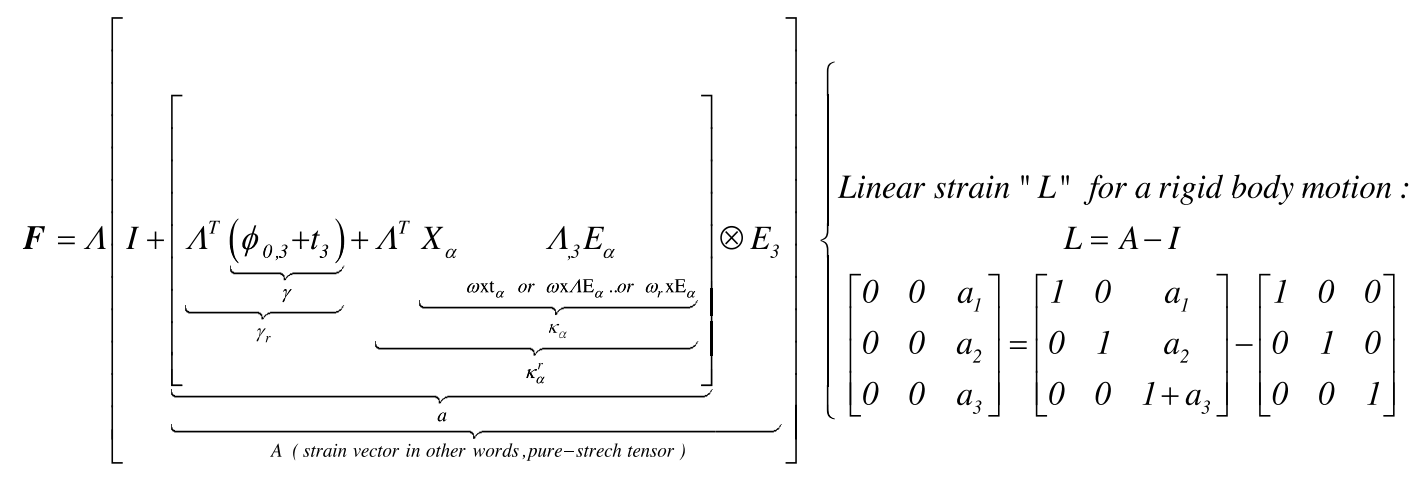

It is noted that linear strain $L$ in Eq. (35) is also utilized for both finite-deformation and small-strain hypotheses models. Particularly, the small-strain hypotheses model for the derivation of FEAP element formulation is preferably utilized assuming approximated Green-Lagrange strain tensor $E^{*}$ as 
$E^{*}=\left(L+L^{T}\right)$

Furthermore, the virtual variations or linearization of the strains $\gamma_{r}$ and $\omega_{r}$ depending on the operator $\aleph_{\delta \Lambda^{T}}($ see a further information about $\aleph_{\delta \Lambda^{T}}$ in Carotenuto, 2006) is written as

$$
\begin{aligned}
& \delta \gamma_{r}=\Lambda^{T} \phi_{0,3}+\aleph_{\delta \Lambda^{T}}\left(\phi_{0,3}\right) \delta \theta \\
& \delta \omega_{r}=T^{T}(\theta) \phi_{0,3}
\end{aligned} \quad\left\{\left\{\begin{array}{c}
\delta \gamma_{r} \\
\delta \omega_{r}
\end{array}\right\}=\left[\begin{array}{ccc}
\Lambda^{T} & \aleph_{\delta A^{T}}\left(\phi_{0,3}\right) & 0 \\
0 & \aleph_{\delta \Lambda^{T}}\left(\theta_{, 3}\right) & T^{T}
\end{array}\right]\left[\begin{array}{cc}
I_{, 3} & 0 \\
0 & I \\
0 & I_{, 3}
\end{array}\right]\left\{\begin{array}{c}
\delta \phi_{0} \\
\delta \theta
\end{array}\right\}\right.
$$

It has to be noted that this linearization in Eq. (37) is accomplished in an indirect approach for the element formulation in the FEAP program (see Carotenuto, 2006). The basic assumptions for the deformation and displacementrelated quantities are given at the preceding part. The stress tensor for the FEAP element can be derived depending on either first Piola-Kirchhoff $P$ or second Piola-Kirchhoff stress tensor $S$. The relation between first and second Piola-Kirchhoff stress tensors is formulated depending on the deformation gradient $\boldsymbol{F}$ :

$$
P=\boldsymbol{F} . S
$$

Thus, the internal work $\delta \Pi_{I n t}$ according to the principle of virtual work is written depending on the linearized strain tensor $\delta a$ (see Eq. (36) and Eq. (37)),

$$
\begin{aligned}
& \Pi_{\text {Int }}=\int_{V} S: \delta E^{*} d V \\
& \Pi_{\text {Int }}=\int_{L}\left[\delta \gamma_{r} \int_{A} S \cdot d A+\delta \omega_{r} \int_{A} X_{\alpha} E_{\alpha} \mathrm{x} S \cdot d A\right] d L \\
& \Pi_{\text {Int }}=\int_{L}\left\{\begin{array}{l}
\delta \gamma_{r} \\
\delta \omega_{r}
\end{array}\right\}\left\{\begin{array}{c}
\int_{A} S \cdot d A \\
\int_{A} X_{\alpha} E_{\alpha} \mathrm{x} S \cdot d A
\end{array}\right\} d L
\end{aligned}
$$

The inner two parts of integration in Eq. (39), briefly $C_{f}$ and $C_{m}$, are written depending on the cross-sectional area $A$, shear module $G$, elasticity module $E$ and the inertia moments $J_{1}, J_{2}, J_{12}$ and $J_{t}$ considering the undeformed configuration of element in the cartesian coordinate:

$$
\begin{aligned}
C_{f} & =\int_{A} S . d A=\left[\begin{array}{ccc}
A G & 0 & 0 \\
0 & A G & 0 \\
0 & 0 & A E
\end{array}\right] \gamma_{r} \\
C_{m} & =\int_{A} X_{\alpha} E_{\alpha} \mathrm{x} S \cdot d A=\left[\begin{array}{ccc}
E J_{1} & E J_{12} & 0 \\
-E J_{21} & E J_{2} & 0 \\
0 & 0 & G J_{t}
\end{array}\right] \omega_{r}
\end{aligned}
$$

Thus, Eq. (39) is rewritten depending on Eq. (37) and Eq. (40-41):

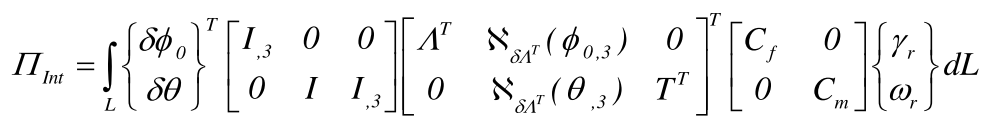

The linearization of Eq. (42) is accomplished: 


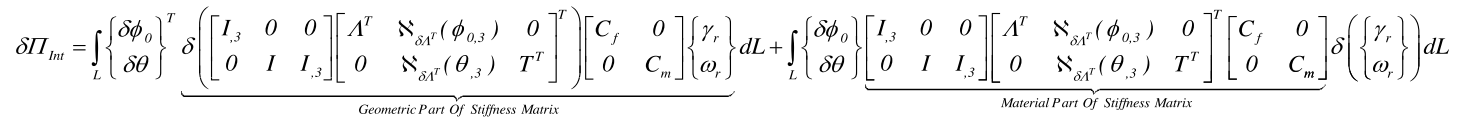

$$
\begin{aligned}
& \delta \Pi_{I n t}=\int_{L}\left\{\begin{array}{c}
\delta \phi_{0} \\
\delta \theta
\end{array}\right\}^{T} \underbrace{(\text { Geometric Part Of Stiffness Matrix }+ \text { Material Part Of Stiffness Matrix })}_{\text {Stiffness Matrix }}\left\{\begin{array}{c}
\delta \gamma_{r} \\
\delta \omega_{r}
\end{array}\right\} d L
\end{aligned}
$$

Furthermore, the external virtual work is written depending on the body forces $b$,

$$
\Pi_{E x t}=\int_{L}\left\{\begin{array}{c}
\delta \phi_{0} \\
\delta \theta
\end{array}\right\}^{T}\left\{\begin{array}{c}
\int_{A}\left[X_{\alpha} t_{\alpha} \mathrm{x} b\right] d A \\
\int_{A}[b] d A
\end{array}\right\} d L
$$

Thus, Eq. (23) is written as

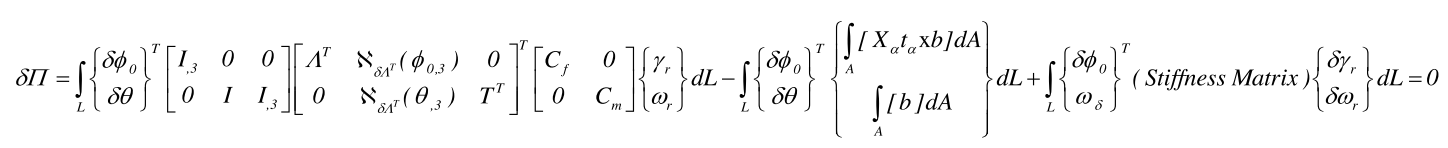

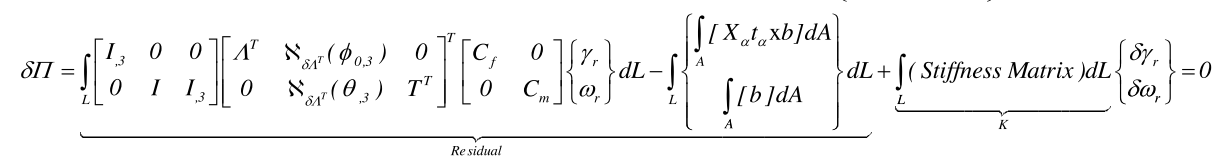

\section{Computing Procedure}

1. Compute the virtual variations of strains $\left\{\begin{array}{l}\delta \gamma_{r} \\ \delta \omega_{r}\end{array}\right\}$ by Eq. (45) as $-K^{-1}$.Residual using the previous iteration (see formfe.for in pmacr2.for along with plsolve in pmacr2.for)

2. Update the position of line centroid, its derivative along with the rotation tensor $\phi_{0}, \phi_{0,3}$ and $\Lambda$ depending on the virtual vwariations of strain $\delta \gamma_{r}$ (see Eq. (35)) (see framf3d.for)

3. Compute the strains $\gamma_{r}$ and $\omega_{r}$ (see Eq. (35)) (see bmshp.for and strefb.for in framf3d.for)

4. Compute the internal and external virtual works $\Pi_{\text {int }}$ and $\Pi_{\text {ext }}$ using Eq. (42) and Eq. (44) (see framf3d.for)

5. Compute the stiffness matrix using Eq. (43) (see geomfb.for and bmbnfb.for in framf3d.for)

6. Compute Residual as $\left(\Pi_{\text {int }}-\Pi_{\text {ext }}\right)$ (see framf $3 d$.for)

\section{THE EMPLOYED NONLINEAR SOLUTION PROCEDURES}

As to the proportional loading, the structural responses are computed, thereby increasing the factored external loading. Then, the incremental load is iteratively controlled depending on the convergence of residual value in order to determine a new incremental load step. This incremental and iterative-based computation begins by computing the residual value as

$$
\mathrm{g}(\mathrm{p}, \lambda)=\mathrm{q}_{\mathrm{i}}(\mathrm{p})-\lambda \mathrm{q}_{\mathrm{ef}}
$$

In Eq. (46), the difference between a "fixed external loading" $q_{e f}$ with factorized by a "load-level parameter" $\lambda$ and "internal forces", $q_{i}$ which varies depending on a "displacement", $p$ is defined by "residual", $g$. Eq. (46) becomes zero at an equilibrium state. The solving of Eq. (46) in a way of using a newton incremental-iterative solution method is formulated as

$$
-\mathrm{K}_{\mathrm{t}} \delta \mathrm{p}=\mathrm{q}_{\mathrm{i}}(\mathrm{p})-\lambda \mathrm{q}_{\mathrm{ef}}
$$


The "iterative displacement", $\delta p$ in Eq. (47) is computed at a new unknown load level $\lambda_{\mathrm{n}}=\lambda_{\mathrm{o}}+\delta \lambda$ and formulated using Eq. (46):

$$
\begin{aligned}
& \delta \mathrm{p}=-\mathrm{K}_{\mathrm{t}}^{-1}\left(\mathrm{q}_{\mathrm{i}}(\mathrm{p})-\lambda \mathrm{q}_{\mathrm{ef}}\right) \\
& =-\mathrm{K}_{\mathrm{t}}^{-1}\left(\mathrm{q}_{\mathrm{i}}\left(\mathrm{p}_{\mathrm{o}}, \lambda_{\mathrm{o}}\right)-\lambda_{\mathrm{n}} \mathrm{q}_{\mathrm{ef}}\right) \\
& =-\mathrm{K}_{\mathrm{t}}^{-1}\left(\mathrm{q}_{\mathrm{i}}\left(\mathrm{p}_{\mathrm{o}}, \lambda_{\mathrm{o}}\right)-\left(\lambda_{\mathrm{o}}+\delta \lambda\right) \mathrm{q}_{\mathrm{ef}}\right) \\
& =-\mathrm{K}_{\mathrm{t}}^{-1}(\underbrace{\left(\mathrm{q}_{\mathrm{i}}\left(\mathrm{p}_{\mathrm{o}}, \lambda_{\mathrm{o}}\right)-\lambda_{\mathrm{of}}\right)}_{\mathrm{g}_{\mathrm{o}}\left(\mathrm{p}_{\mathrm{o}}, \lambda_{\mathrm{o}}\right)}-\delta \lambda \mathrm{q}_{\mathrm{ef}}) \\
& =-\underbrace{\mathrm{K}_{\mathrm{t}}^{-1} \mathrm{~g}_{\mathrm{o}}}_{\delta \mathrm{p}}+\delta \lambda \underbrace{\mathrm{K}_{\mathrm{t}}^{-1} \mathrm{q}_{\mathrm{ef}}}_{\delta \mathrm{p}_{\mathrm{t}}}=\delta \overline{\mathrm{p}}+\delta \lambda \delta \mathrm{p}_{\mathrm{t}}
\end{aligned}
$$

Thus, a new incremental displacement is obtained using Eq. (48):

$$
\Delta \mathrm{p}_{\mathrm{n}}=\Delta \mathrm{p}_{\mathrm{o}}+\delta \mathrm{p}=\Delta \mathrm{p}_{\mathrm{o}}+\delta \overline{\mathrm{p}}+\delta \lambda \delta \mathrm{p}_{\mathrm{t}}
$$

However, "incremental load parameter" $\delta \lambda$ is still unknown. For this purpose, an "arc-length" $\Delta l$ is specified at an equilibrium point $\left(p_{o}, \lambda_{o} q_{e f}\right)$ and aimed to intersect with the load-displacement path (see the derivation of arc-length in Crisfield (1997b)). The "arc-length" $s$ is formulated as

$$
\mathrm{ds}=\sqrt{\left(\mathrm{dp} p^{\mathrm{T}} \mathrm{dp}+\mathrm{d} \lambda \varphi^{2} \mathrm{q}_{\mathrm{ef}}^{\mathrm{T}} \mathrm{q}_{\mathrm{ef}}\right)}
$$

The arc length in Eq. (50) is in an incremental form:

$$
\mathrm{a}=\left(\Delta \mathrm{p}^{\mathrm{T}} \Delta \mathrm{p}+\Delta \lambda^{2} \varphi^{2} \mathrm{q}_{\mathrm{ef}} \mathrm{q}_{\mathrm{ef}}\right)-\Delta l^{2}=0
$$

Eq. (51) is utilized to derive a number of variations of arc-length techniques. Therefore, Eq. (51) is firstly used to derive the "spherical arc-length technique". For this purpose, Eq. (51) for both "new" $n$ and "old" $o$ iterations is rewritten as

$$
\Delta l^{2}=\left(\Delta \mathrm{p}_{\mathrm{o}}^{\mathrm{T}} \Delta \mathrm{p}_{\mathrm{o}}+\Delta \lambda_{\mathrm{o}}^{2} \varphi^{2} \mathrm{q}_{\mathrm{ef}}^{\mathrm{T}} \mathrm{q}_{\mathrm{ef}}\right)=\left(\Delta \mathrm{p}_{\mathrm{n}}^{\mathrm{T}} \Delta \mathrm{p}_{\mathrm{n}}+\Delta \lambda_{\mathrm{n}}^{2} \varphi^{2} \mathrm{q}_{\mathrm{ef}}^{\mathrm{T}} \mathrm{q}_{\mathrm{ef}}\right)
$$

In order to obtain $\delta \lambda$ depending on a "scaling parameter" $\varphi, \Delta p_{n}$ in Eq. (52) is introduced to Eq. (52). Then, the quadratic equation in Eq. (52) using Eq. (51) is solved for $\delta \lambda$.

$$
\left.\begin{array}{l}
\mathrm{a}_{1} \delta \lambda^{2}+\mathrm{a}_{2} \delta \lambda+\mathrm{a}_{3}=0 \\
\left.\mathrm{a}_{1}=\delta \mathrm{p}_{\mathrm{t}}^{\mathrm{T}} \delta \mathrm{p}_{\mathrm{t}}+\varphi^{2} \mathrm{q}_{\mathrm{ef}}^{\mathrm{T}} \mathrm{q}_{\mathrm{ef}}\right) \\
\mathrm{a}_{2}=2 \delta \mathrm{p}_{\mathrm{t}}\left(\Delta \mathrm{p}_{\mathrm{o}}+\delta \overline{\mathrm{p}}\right)+2 \Delta \lambda_{\mathrm{o}} \varphi^{2} \mathrm{q}_{\mathrm{ef}}^{\mathrm{T}} \mathrm{q}_{\mathrm{ef}} \\
\mathrm{a}_{3}=\left(\Delta \mathrm{p}_{\mathrm{o}}+\delta \overline{\mathrm{p}}\right)^{\mathrm{T}}\left(\Delta \mathrm{p}_{\mathrm{o}}+\delta \overline{\mathrm{p}}\right)-\Delta l^{2}+\Delta \lambda_{\mathrm{o}}^{2} \varphi^{2} \mathrm{q}_{\mathrm{ef}}^{\mathrm{T}} \mathrm{q}_{\mathrm{ef}}
\end{array}\right\}
$$

In this regard, Eq. (51) can be utilized to derive "linearized arc-length" technique as well. Thus, Eq. (51) is rewritten using Taylor series:

$$
\mathrm{a}_{\mathrm{n}}=\mathrm{a}_{\mathrm{o}}+2 \Delta \mathrm{p}^{\mathrm{T}} \partial \mathrm{p}+2 \Delta \lambda \partial \lambda \varphi^{2} \mathrm{q}_{\mathrm{ef}} \mathrm{q}_{\mathrm{ef}}=0
$$

Eq. (55) is rewritten for the term $a_{0}$ : 


$$
\left.-\mathrm{a}_{\mathrm{o}} / 2=2 \Delta \mathrm{p}_{\mathrm{o}}^{\mathrm{T}} \partial \mathrm{p}+2 \Delta \lambda_{\mathrm{o}} \partial \lambda \varphi^{2} \mathrm{q}_{\mathrm{ef}}^{\mathrm{T}} \mathrm{q}_{\mathrm{ef}}\right)
$$

If $\mathrm{a}_{\mathrm{o}}$ is taken as zero, the "iterative change" $\left(\delta p, \delta \lambda \varphi q_{e f}\right)$ becomes orthogonal to the "secant change" $\left(\Delta p_{o}, \Delta \lambda_{o} \varphi q_{e f}\right)$

If "iterative displacement" $\delta p$ in Eq. (48) is introduced to Eq. (56), "incremental load parameter" $\delta \lambda$ is obtained using "linearized arc-length" technique:

$$
\delta \lambda\left(\Delta \mathrm{p}_{\mathrm{o}}, \Delta \lambda_{\mathrm{o}}\right)=\frac{-\Delta \mathrm{p}_{\mathrm{o}}^{\mathrm{T}} \partial \overline{\mathrm{p}}}{\Delta \mathrm{p}_{\mathrm{o}} \delta \mathrm{p}_{\mathrm{t}}+\Delta \lambda_{\mathrm{o}} \varphi^{2} \mathrm{q}_{\mathrm{ef}}^{\mathrm{T}} \mathrm{q}_{\mathrm{ef}}}
$$

The computing complexity of linearized arc-length technique is lower than the spherical arc-length technique because of not requiring solving any algebraic equation and choosing the correct root. Therefore, it is preferably improved by implementation of available iterative search techniques. One of the improvements is the hybridization of a line search technique with linearized arc-length technique. In this regard, the arc length, which is formulated in Eq. (55) using Taylor series and utilized to derive the linearized arc-length technique, is employed to hybridize the arclength technique with a line search technique. One of these attempts is based on taking the value of scaling parameter $\varphi$ to be zero. Thus, Eq. (55) is rewritten using the iterative displacement, $\delta p$ :

$$
\Delta \mathrm{p}^{\mathrm{T}} \partial \mathrm{p}=\Delta \mathrm{p}^{\mathrm{T}}\left(\overline{\delta \mathrm{p}}+\delta \lambda \delta \mathrm{p}_{\mathrm{t}}\right)=0
$$

Thus, the incremental load parameter, $\delta \lambda$ becomes the following form:

$$
\delta \lambda=\frac{-\Delta \mathrm{p}_{\mathrm{o}}^{\mathrm{T}} \partial \stackrel{-}{\mathrm{p}}}{\Delta \mathrm{p}_{\mathrm{o}} \delta \mathrm{p}_{\mathrm{t}}}
$$

The implementation of line-search technique in the linearized arc-length technique, which is assumed to be $\varphi=0$, is constituted on a multiplying of iterative disp. $\delta p$ by a "line search step length" $\eta$. In this regard, the linearized and spherical arc-length techniques are utilized by Opensees (see ArcLength.cpp and ArcLength1.cpp). FEAP uses the linearized arc-length approach hybridized by the line-search technique (see Figure 1) (see arclen.for).

Linearized and spherical arc-lengths are utilized by FEAP and OpenSees programs in order to solve the nonlinear structural responses under the predefined loading conditions. In order to introduce their governing parameters utilized by FEAP and OpenSees programs, a typical pseudocode is presented in Figure 1. 
Predefine "Number of Increment", "Number of Iteration", "Convergence Tolerance" tol, Fixed External Load $q_{e f}$ Predefine first adjustment $\left\{\begin{array}{l}\text { "Arc Length" } \Delta p_{o}^{T} \partial \bar{p} \text {, "Scaling Parameter or Alpha" } \varphi \text { for OpenSees } \\ \text { "Convergence Tolerance for Residual" for FEAP }\end{array}\right.$

Assume $p_{o}=0, \lambda=0, \delta p=q_{e f}$,

for $i=1$ :Number of Increment

Predictor Step

form element and system stiffness matrix, $K_{t}, K_{T}$

Solve $\delta p_{t}=K_{T}^{-1} \cdot q_{e f}$ and $\delta \bar{p}=K_{T}^{-1} \cdot g_{o}$

if $i=1$, Compute $\delta \lambda_{0}$ using $\left\{\begin{array}{ll}E q .57 & \text { for OpenSees } \\ E q .59 & \text { for FEAP }\end{array}\right.$, end

for $j=1$ :Number of Iteration

Corrector Step

if $j=1, \delta \lambda=\delta \lambda_{0}$, else, $\delta \lambda_{0}=\delta \lambda$ end

Solve $\delta p_{t}=K_{T}^{-1} \cdot q_{e f}$ and $\delta \bar{p}=K_{T}^{-1} \cdot g_{o}$

Assume $\eta=1$,

Compute $\delta \lambda$ using $\left\{\begin{array}{l}E q .56 \quad \text { if linearized arc length utilized for OpenSees } \\ E q .53 \text { and } E q .54 \quad \text { if spherical arc length utilized for OpenSees } \\ E q .59 \quad \text { for FEAP }\end{array}\right.$

if $\delta \lambda_{o} \geq$ tol $r a t=\frac{\delta \lambda}{\delta \lambda_{o}}$, else, $r a t=\frac{\delta \lambda}{\delta \lambda_{o}+t o l}$, end $\quad$ for only FEAP

if $((-1 \leq r a t$ and $r a t \leq 0.0), \eta=0.5$, end for only FEAP

Update $\lambda=\delta \lambda . \eta+\lambda$ and $p=p_{o}+\left(\delta p_{t}+\delta \bar{p}\right) \cdot \eta$

end

if convergence (tol) is satisfied, STOP

end

Figure 1. A Pseudocode for The Use of Arc-length Techniques

\section{APPLICATION EXAMPLES}

In this section, the postbuckling analyses of six benchmark problems are performed using two different element approaches and nonlinear solution techniques. Thus, it is possible to compare the computing efficiencies of the employed solution procedures with respect to the employed elements. The computing performances are evaluated depending on the two different elements-related parameters, the number of element and integration points. Furthermore, the nonlinear solutions techniques have a big sensitivity to any variation in their governing parameters. Therefore, the better combinations of governing parameters, number of increment "NIN", and number of iteration "NIT" for FEAP along with arc-length value "ALV" and alpha value "AV" for Opensees are obtained from a number of nonlinear structural analysis trials and presented for each benchmark problems (see Table 1). It is noted that the parameter $A V$ equals zero for the use of linearized arc-length technique. For this purpose, two available software devices with 
open-source code named Opensees and FEAP are utilized for the computation of the nonlinear structural analyses. It is noted that the relatively higher converged solutions are obtained from the trials with the parameter combinations of higher elements and/or integration points for both Opensees and FEAP. In order to make a further observation in the nonlinear analysis of structural systems, a movie for each of six benchmark tests with the decreased elements is also included for both Opensees and FEAP, respectively. It is noted that the computing performances of Opensees and FEAP are easily affected from minor adjustments of their governing parameters.

The shear modulus $G$ is assumed to be computed depending on the elasticity modulus $E$ and Poisson ratio $v$ as $G=\frac{E}{2 *(I+v)}$. The shear correction factor is taken as $(5 / 6)$.

While the governing parameters of Opensees and FEAP are listed for six benchmark problems in Table 1, the elapsed times during execution of Opensees and FEAP are summarized in Table 2.

Table 1. The Main Governing Parameter Values of Employed Solution Algorithms and Elements.

\begin{tabular}{|c|c|c|c|c|c|c|c|c|c|c|c|}
\hline \multirow[t]{2}{*}{ Example } & \multirow[t]{2}{*}{ ENSA } & \multicolumn{4}{|c|}{$\begin{array}{c}\text { Governing Parameters of } \\
\text { ENSA }\end{array}$} & \multicolumn{4}{|c|}{$\begin{array}{l}\text { Governing Parameters } \\
\text { of Convergence Tests }\end{array}$} & \multicolumn{2}{|c|}{$\begin{array}{c}\text { Governing } \\
\text { Parameters } \\
\text { of Employed } \\
\text { Elements }\end{array}$} \\
\hline & & NIN & NIT & ALV & AV & $\begin{array}{c}\mathrm{CT} \\
\text { (Energy) }\end{array}$ & MNIT & $\begin{array}{c}\mathrm{CT} \\
\text { (Residual) }\end{array}$ & $\begin{array}{l}\text { Zero } \\
\text { Energy }\end{array}$ & NIT & CT \\
\hline \multirow{2}{*}{$\begin{array}{l}\text { Cantilever } \\
\text { Beam }\end{array}$} & Opensees & 500000 & 10 & 0.015 & 0.01 & $1.0 \mathrm{e}-25$ & 10 & - & - & 100 & $1.0 \mathrm{e}-25$ \\
\hline & FEAP & 5000 & 10 & - & - & $\begin{array}{c}1.0 \mathrm{e}-16 \\
\text { (default) }\end{array}$ & - & $\begin{array}{l}1.0 \mathrm{e}-8 \\
\text { (default) }\end{array}$ & 0.00 & - & - \\
\hline \multirow{2}{*}{$\begin{array}{l}\text { Portal } \\
\text { Frame }\end{array}$} & Opensees & 70000 & 10 & 0.01 & 0.0001 & $1.0 \mathrm{e}-15$ & 10 & - & - & 100 & $1.0 \mathrm{e}-25$ \\
\hline & FEAP & 20000 & 10 & - & - & $\begin{array}{c}1.0 \mathrm{e}-16 \\
\text { (default) }\end{array}$ & - & $\begin{array}{l}1.0 \mathrm{e}-8 \\
\text { (default) }\end{array}$ & 0.00 & - & - \\
\hline \multirow{2}{*}{$\begin{array}{l}\text { Simply } \\
\text { Beam }\end{array}$} & Opensees & 50000 & 10 & 0.001 & 0.001 & $1.0 \mathrm{e}-25$ & 10 & - & - & 100 & $1.0 \mathrm{e}-25$ \\
\hline & FEAP & 2800 & 10 & - & - & $\begin{array}{c}1.0 \mathrm{e}-16 \\
\text { (default) }\end{array}$ & - & $\begin{array}{l}1.0 \mathrm{e}-8 \\
\text { (default) }\end{array}$ & 0.00 & - & - \\
\hline \multirow{2}{*}{$\begin{array}{l}\text { William } \\
\text { Toggle }\end{array}$} & Opensees & 50000 & 10 & 0.001 & 0.01 & $1.0 \mathrm{e}-25$ & 10 & - & - & 100 & $1.0 \mathrm{e}-25$ \\
\hline & FEAP & 4000 & 10 & - & - & $\begin{array}{c}1.0 \mathrm{e}-16 \\
\text { (default) }\end{array}$ & - & $\begin{array}{l}1.0 \mathrm{e}-8 \\
\text { (default) }\end{array}$ & 0.00 & - & - \\
\hline \multirow[b]{2}{*}{ Arch } & Opensees & 70000 & 10 & 0.1 & 0.01 & $1.0 \mathrm{e}-15$ & 10 & - & - & 100 & $1.0 \mathrm{e}-25$ \\
\hline & FEAP & 1000 & 10 & - & - & $\begin{array}{c}1.0 \mathrm{e}-16 \\
\text { (default) }\end{array}$ & - & $\begin{array}{l}1.0 \mathrm{e}-8 \\
\text { (default) }\end{array}$ & 0.00 & - & - \\
\hline \multirow{2}{*}{$\begin{array}{l}\text { Lee } \\
\text { Frame }\end{array}$} & Opensees & 80000 & 10 & 0.1 & 0.01 & $1.0 \mathrm{e}-15$ & 10 & - & - & 100 & $1.0 \mathrm{e}-25$ \\
\hline & FEAP & 2000 & 10 & - & - & $\begin{array}{c}1.0 \mathrm{e}-16 \\
\text { (default) }\end{array}$ & - & $\begin{array}{l}1.0 \mathrm{e}-8 \\
\text { (default) }\end{array}$ & 0.00 & - & - \\
\hline
\end{tabular}


Table 2. The Elapsed Time for Execution of Employed Nonlinear Solution algorithms.

\begin{tabular}{|c|c|c|c|c|c|c|c|}
\hline \multirow[t]{2}{*}{ Example } & \multirow[t]{2}{*}{ ENSA } & \multicolumn{6}{|c|}{$\begin{array}{l}\text { Elapsed Time for The Execution of Employed Nonlinear Solution algorithms } \\
\text { Using Different Parameter Sets Utilized in Element Formulation }\end{array}$} \\
\hline & & $\begin{array}{c}\mathrm{DN}=4 \& \\
\mathrm{NIP}=2\end{array}$ & $\begin{array}{l}\mathrm{DN}=4 \& \\
\mathrm{NIP}=10\end{array}$ & $\begin{array}{l}\mathrm{DN}=10 \& \\
\mathrm{NIP}=2\end{array}$ & $\begin{array}{c}\mathrm{DN}=10 \& \\
\mathrm{NIP}=10\end{array}$ & $\mathrm{DN}=4$ & $\mathrm{DN}=10$ \\
\hline \multirow{2}{*}{$\begin{array}{c}\text { Cantilever } \\
\text { Beam }\end{array}$} & Opensees & 1.8942 & 1.6719 & 5.4098 & 6.2725 & - & - \\
\hline & FEAP & - & - & - & - & 24.0004 & 27.5644 \\
\hline \multirow{2}{*}{$\begin{array}{l}\text { Portal } \\
\text { Frame }\end{array}$} & Opensees & 80.5330 & 81.7293 & 160.4939 & 159.5100 & - & 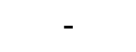 \\
\hline & FEAP & - & - & - & - & 101.3010 & 131.4181 \\
\hline \multirow{2}{*}{$\begin{array}{l}\text { Simply } \\
\text { Beam }\end{array}$} & Opensees & 33.2670 & 33.4963 & 46.7105 & 46.9425 & - & - \\
\hline & FEAP & - & & - & - & 13.3570 & 15.3948 \\
\hline \multirow{2}{*}{$\begin{array}{l}\text { William } \\
\text { Toggle }\end{array}$} & Opensees & 30.5461 & 30.4222 & 34.798 & 34.3699 & - & - \\
\hline & FEAP & - & - & - & - & 22.5353 & 27.8520 \\
\hline \multirow{2}{*}{ Arch } & Opensees & 20.3706 & 20.1530 & 429.0021 & 429.2157 & - & - \\
\hline & FEAP & - & - & - & - & 5.5707 & 6.0546 \\
\hline \multirow{2}{*}{$\begin{array}{c}\text { Lee } \\
\text { Frame }\end{array}$} & Opensees & 15.8066 & 15.9966 & 86.2060 & 84.8022 & - & - \\
\hline & FEAP & - & - & - & - & 11.6716 & 13.8712 \\
\hline
\end{tabular}

ENSA: Employed Nonlinear Solution Approaches; DN: Division Number; NIP: Number of Integral Point

\subsection{Cantilever Beam Subjected to a Bending Moment}

This example is a cantilever beam with a length of $10 \mathrm{~m}$ subjected to a concentrated moment with a magnitude of $10 \mathrm{kN} . \mathrm{m}$ at the free end as shown in Figure 2 (Hsiao and Huo, 1987). The elasticity module and Poison Ratio are $120000 \mathrm{kN} / \mathrm{m}^{2}$ and 0.3 . The cross-section of beam is rectangular with a dimension $1.0 \mathrm{mx} 0.1 \mathrm{~m}$. The main feature of this beam is its ability of bending into a full circle. The vertical and horizontal displacements of free end are displayed for both Opensees and FEAP in Figures 3-4.

The relatively higher converged solution of this cantilever beam is obtained by Opensees. The success of linearized arc-length method compared to the spherical arc-length one for Opensees is easily observed from the almost full tracing of equilibrium curve (see Figure 3a-3b and Figure 4a-4b). The linearized arc-length method utilized in Opensees is not affected from an increase in the number of element and integral points (see Figure 3a and Figure 4a). The similar result is also valid for using the increased element number for FEAP (see Figures $3 \mathrm{c}$ and $4 \mathrm{c}$ ). The success of linearized arc-length method for Opensees is higher than that of linearized arc-length method for FEAP (consider the complete curve in Figure 3c and Figure 4c). However, the elapsed time for the execution of FEAP is lower than that of Opensees (see Table 2).

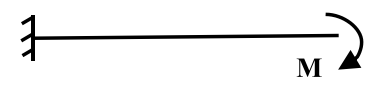

Figure 2. Cantilever Beam. 

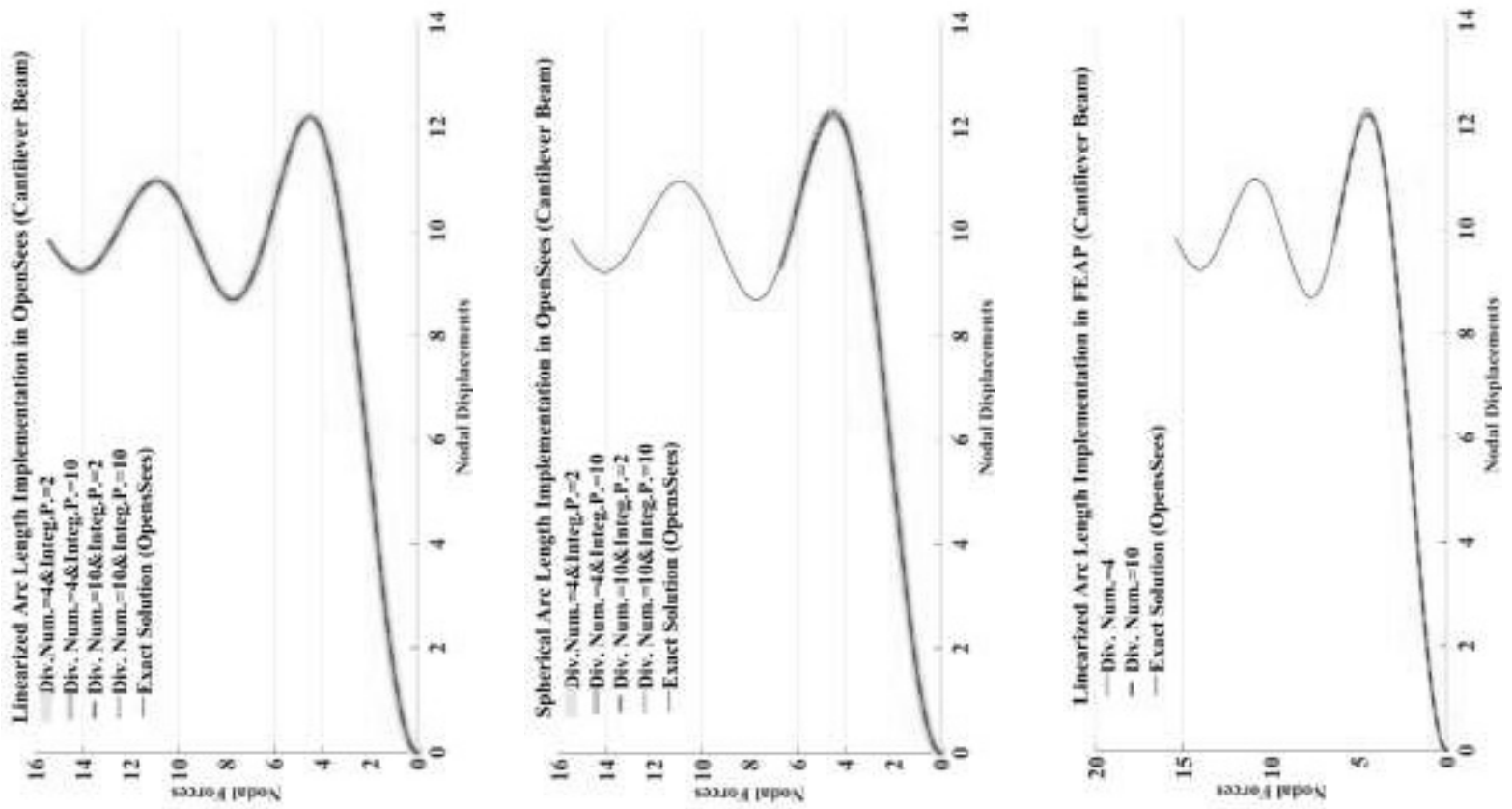

Figure 3. Comparisons of Linearized and Spherical Arc Length Method with an implementation in Opensees along with Spherical Arc Length Method with an implementation in FEAP.
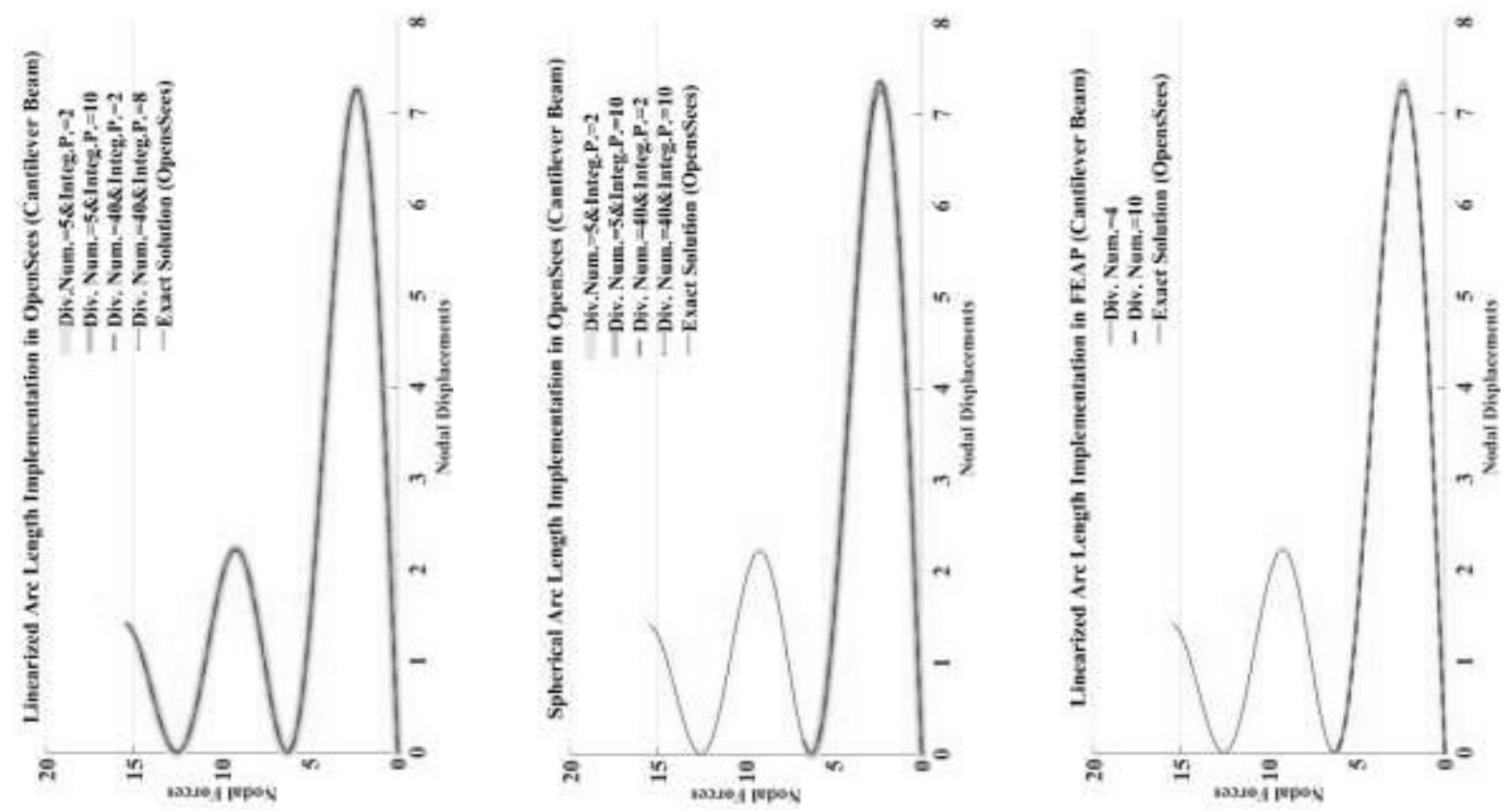

Figure 4. Comparisons of Linearized and Spherical Arc Length Method with an implementation in Opensees along with Spherical Arc Length Method with an implementation in FEAP. 


\subsection{Simply Supported Beam Subjected to a Concentrated and Axial Load}

This Euler type beam, which is one of the classic nonlinear examples, is subjected to both axial and middle loads (Hsiao and Huo, 1987 and Shames and Dym, 1985). The magnitudes of axial and middle loads are $30 \mathrm{kN}$ and 0.072/30 $\mathrm{kN}$ (see Figure 5). While the length of beam is $10 \mathrm{~m}$, the cross-sectional area and inertia moment are taken as $1 \mathrm{~m}^{2}$ and $0.0001 \mathrm{~m}^{4}$. The elasticity module and poison ratio of the beam material are $1000000 \mathrm{kN} / \mathrm{m}^{2}$ and 0.3 . Whereas the relatively higher converged solution, which shows the middle vertical displacement and load response, is obtained by FEAP (see the small Figure in Figure 6c), the linearized and spherical arc-length methods of Opensees along with linearized arc-length method of FEAP are displayed for different numbers of elements and integration points in Figure $6 \mathrm{a}$ and $6 \mathrm{~b}$. Although the coarse (4 elements) or fine mesh (10 elements) along with lower (2 integration points) or higher (10 integration points) integration has not any effect on the predicting the full tracing of equilibrium curve, the linearized arc-length methods of Opensees become more successful then spherical one (see Figure 6a and 6b). An increase in the number of elements in FEAP leads to an elevation in the accurateness degree for predicting a correct equilibrium curve (see Figure 6c). Moreover, the accurateness degree of linearized arc-length method of FEAP in describing postbuckling behavior of the beam is higher compared to the linearized one of Opensees (see Figure $6 \mathrm{~b}$ and 6c). However, the elapsed time for the execution of FEAP is lower than Opensees (see Table 2).

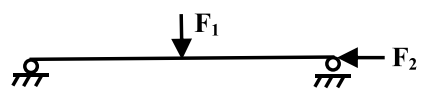

Figure 5. Simply Supported Beam.
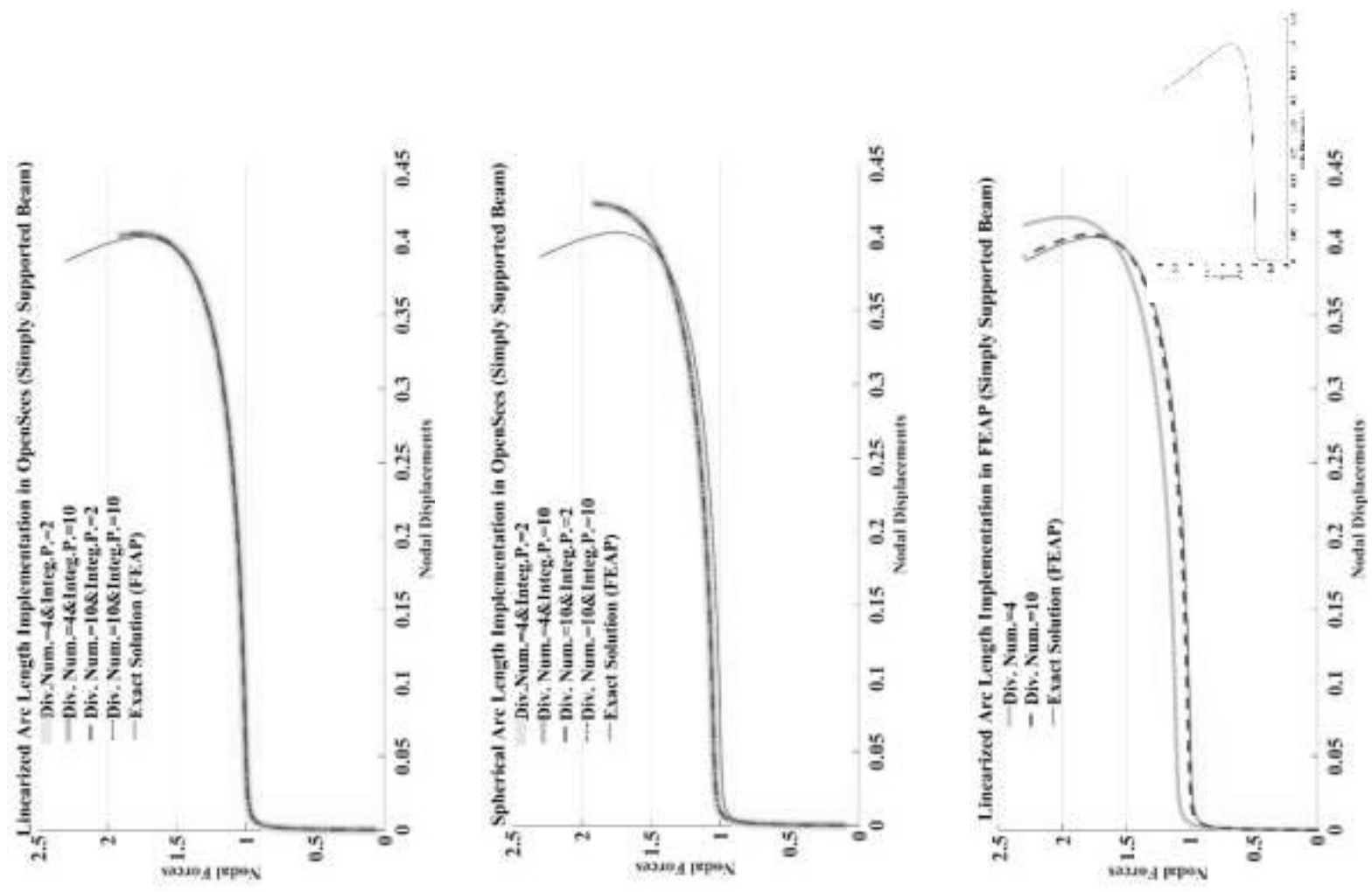

Figure 6. Comparisons of Linearized and Spherical Arc Length Method with an implementation in Opensees along with Spherical Arc Length Method with an implementation in FEAP. 


\subsection{Clamped Hinged-Deep Arch Subjected to a Concentrated Load}

The crown of this deep arch with a degree of 215 and a radius of $500 \mathrm{~cm}$ is subjected to a single vertical load of $100 \mathrm{~N}$ (Hsiao and Huo, 1987 and Deppo and Schmidt, 1975). The elasticity module and poison ratio of the beam material are taken as $200000 \mathrm{~N} / \mathrm{cm}^{2}$ and 0.3 (see Figure 7). The cross-sectional area and inertia moment are assumed as $100 \mathrm{~cm}^{2}$ and $833 \mathrm{~cm}^{4}$. The variation of (Load*Radius $\left.{ }^{2}\right) /($ Elasticity Module*Radius) with (Vertical and Horizontal Displacement of Crown/Radius) is displayed for both Opensees and FEAP in Figures 8 and 9. The relatively higher converged solution of this cantilever beam is obtained by FEAP. The success of linearized arc-length method compared to the spherical arc-length one for Opensees is easily observed from the almost full tracing of equilibrium curve (see Figure $8 \mathrm{a}-8 \mathrm{~b}$ and Figure $9 \mathrm{a}-9 \mathrm{~b})$. The linearized arc-length method utilized in Opensees is not affected from an increase in the number of element and integral points (see Figure 8a and Figure 9a). An increase in the number of elements in FEAP leads to an elevation in the accurateness degree for predicting a correct equilibrium curve (see Figures $8 \mathrm{c}$ and 9c). Moreover, the accurateness degree of linearized arc-length method of FEAP in describing postbuckling behavior of the beam is higher compared to the linearized one of Opensees (see Fig. $8 \mathrm{~b}$ and $9 \mathrm{c}$ ). However, the elapsed time for the execution of FEAP is lower than that for Opensees (see Table 2).

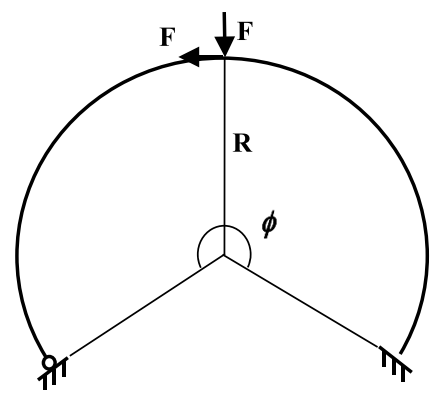

Figure 7. Clamped-hinged deep arch.
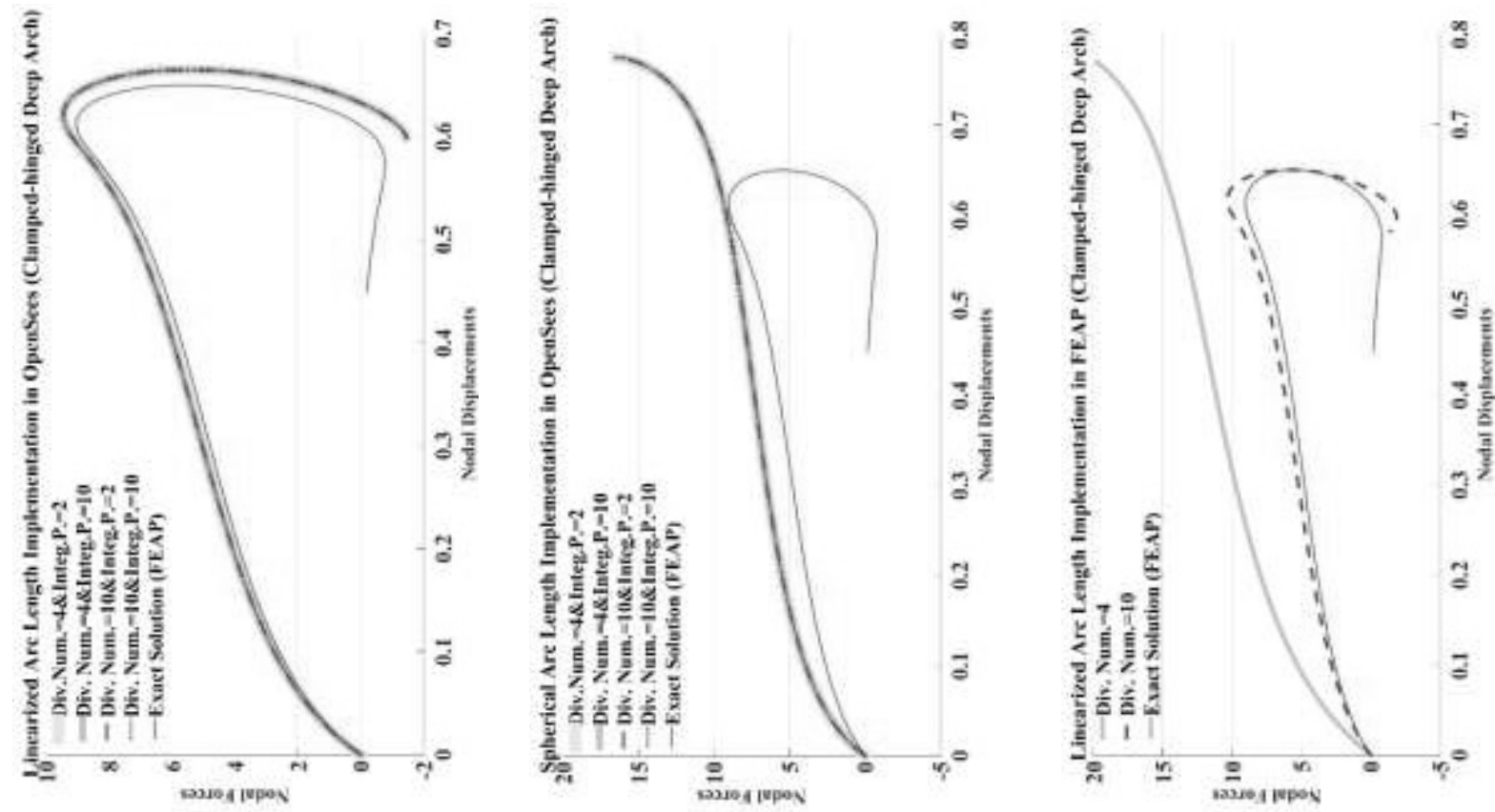

Figure 8. Comparisons of Linearized and Spherical Arc Length Method with an implementation in Opensees along with Spherical Arc Length Method with an implementation in FEAP. 

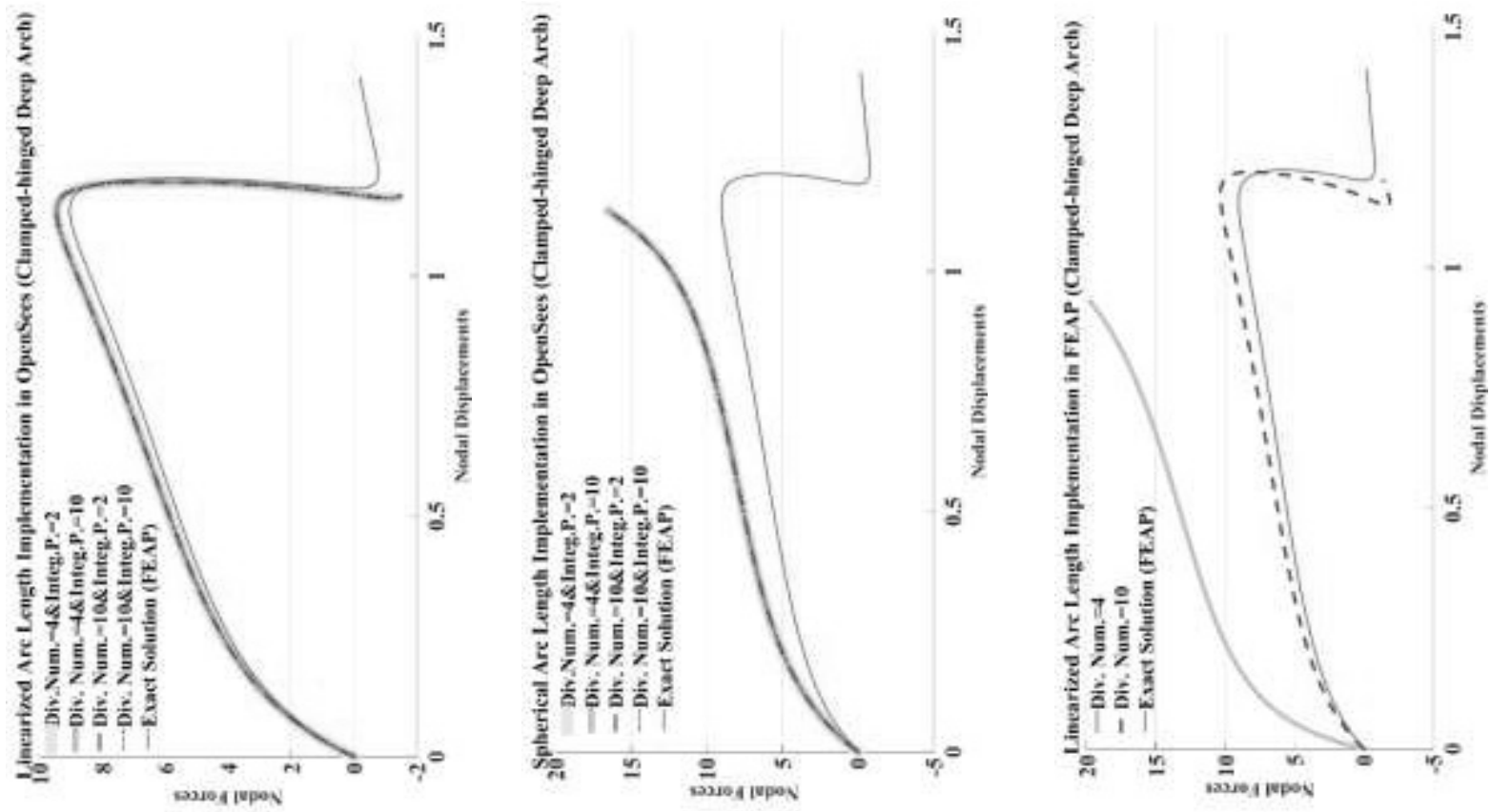

Figure 9. Comparisons of Linearized and Spherical Arc Length Method with an implementation in Opensees along with Spherical Arc Length Method with an implementation in FEAP.

\subsection{Williams Toggle Frame Subjected to Concentrated Loads}

This toggle frame in Figure 10 is firstly devised by Williams (1964). It is also investigated by Hsiao and Huo, 1987, Wood and Zienkiewicz, 1977, Pacoste and Eriksson, 1997 and Nanakorn and Vu, 2006. The toggle frame has a middle height of $0.386 \mathrm{in}$. and span of $12.943 \mathrm{in.}$ The form of cross-sectional area is rectangular with a dimension of $0.753 \mathrm{in}$ and $0.243 \mathrm{in}$. The elasticity module and poison ratio of the frame material are $10300000 \mathrm{lb} / \mathrm{in}^{2}$ and 0.3 . It is subjected to a middle load of $80 \mathrm{lb}$. The load and vertical-horizontal displacement curves are computed and plotted in Figures 11 and 12. The relatively higher converged solution, which shows the middle vertical displacement and load response, is obtained by FEAP (see the small Figures in Figures 11c and 12c). Whereas the coarse (4 elements) or fine mesh (10 elements) along with lower ( 2 integration points) or higher (10 integration points) integration for Opensees has not any effect on predicting the full tracing of equilibrium curve (see Figure 11 (a-b) and 12 (a-b)), the fine mesh for FEAP becomes important for a higher prediction (see Figure 11c). The linearized arc-length method for FEAP is exhibited more accurately than linearized arc-length method for Opensees (consider the equilibrium curves in Figures 11a, 12a and 11c, 12c). The elapsed time for the execution of FEAP is lower than Opensees (see Table 2).

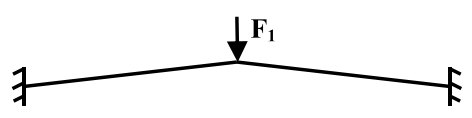

Figure 10. Williams Toggle Frame. 

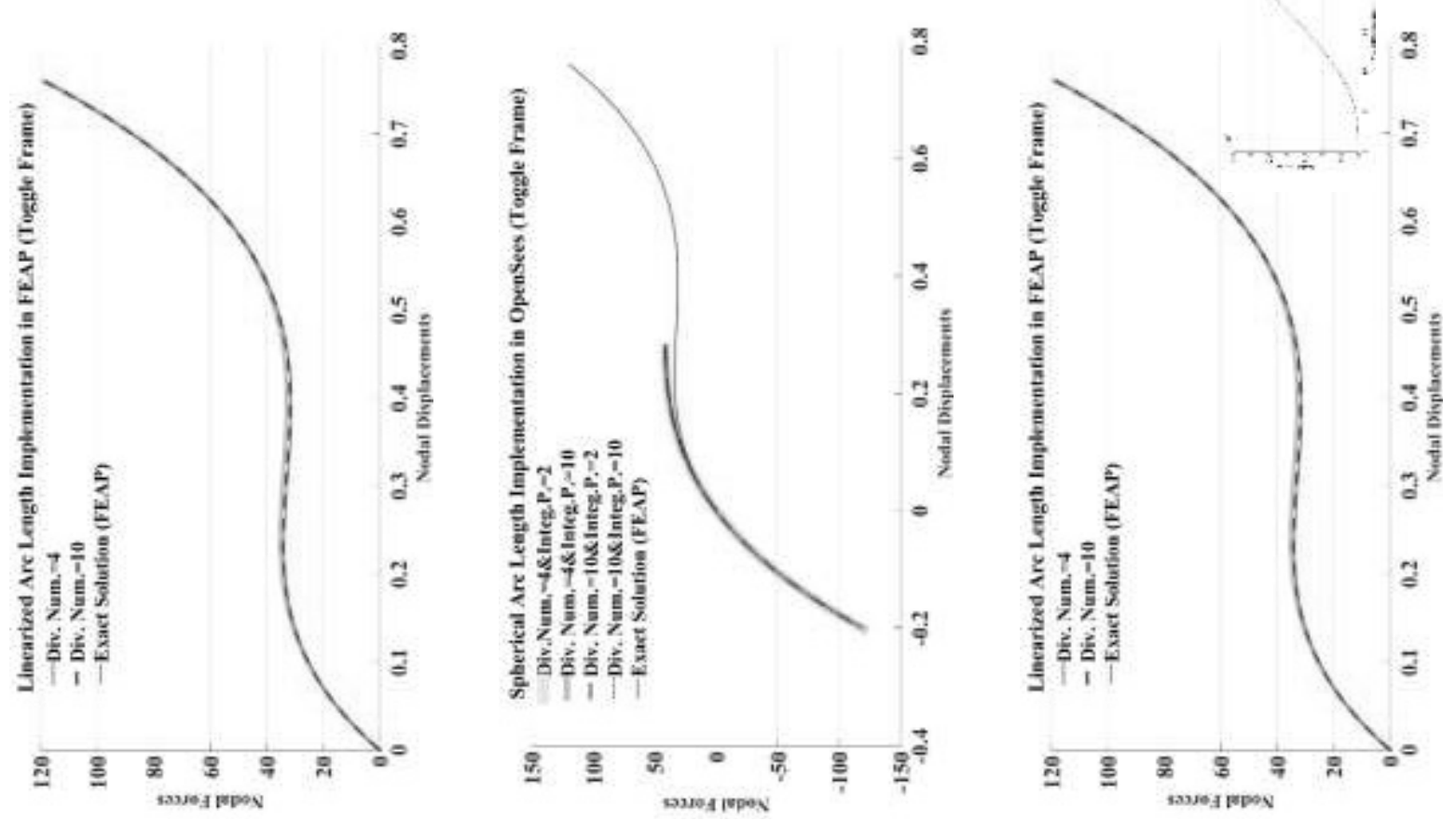

Figure 11. Comparisons of Linearized and Spherical Arc Length Method with an implementation in Opensees along with Spherical Arc Length Method with an implementation in FEAP.
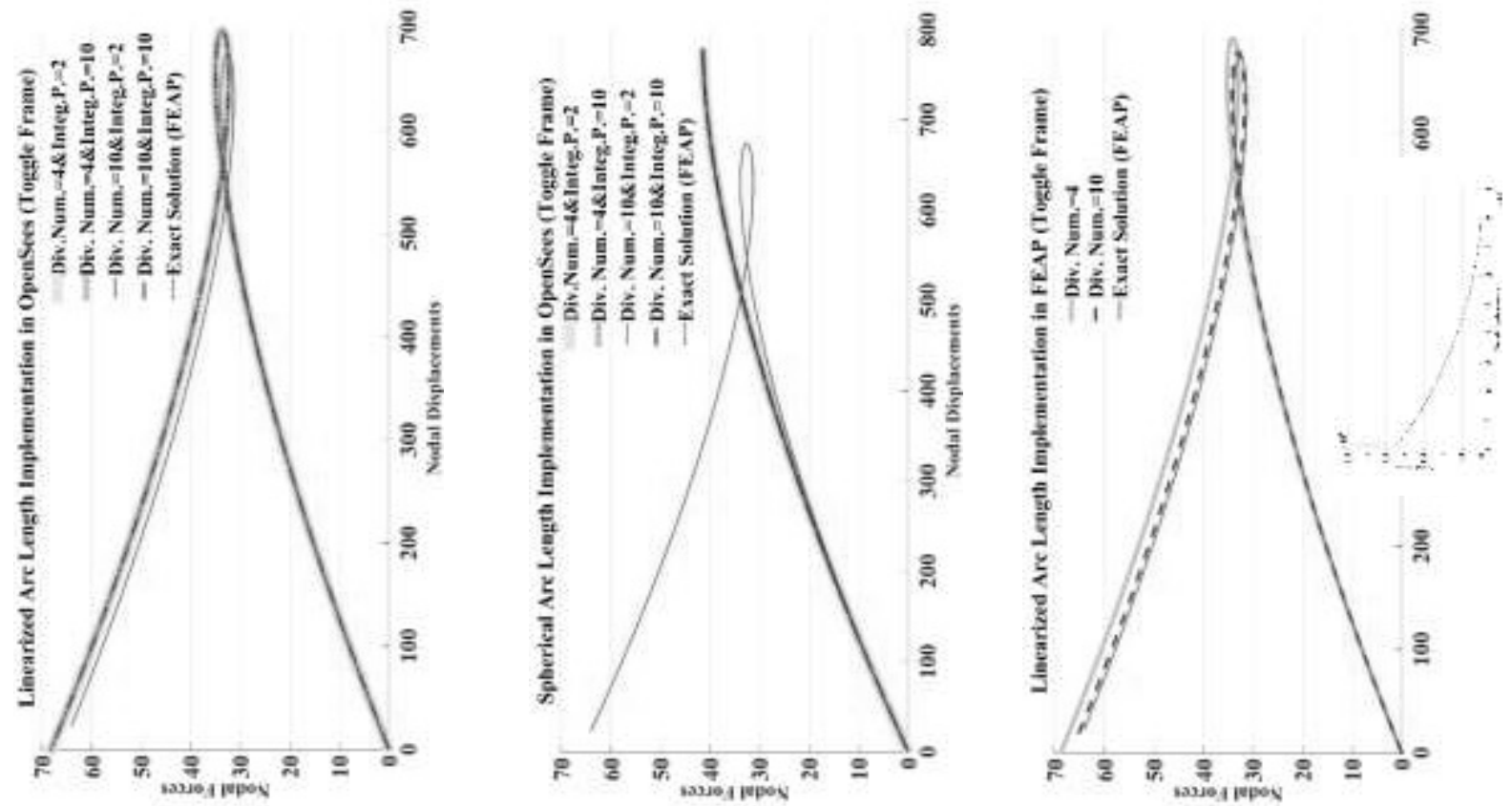

Figure 12. Comparisons of Linearized and Spherical Arc Length Method with an implementation in Opensees along with Spherical Arc Length Method with an implementation in FEAP. 


\subsection{Lee Frame Subjected to a Concentrated Load}

This well-known classic nonlinear benchmark test under a concentrated load of $100 \mathrm{kN}$ shown in Figure 13 has been firstly investigated by Lee et al. (1968). Then, it has subsequently been examined by Pacoste and Eriksson (1997), Hsiao and Huo (1987), Cichon (1984), Smolenski (1999), and Planinc and Saje (1999). The concentrated load is located on the fifth segment of the upper bar. This asymmetric frame, each part of which has a length of $120 \mathrm{~cm}$, has a material, elasticity module, and poison ratio, which are $200000 \mathrm{kN} / \mathrm{cm}^{2}$ and 0.3 . The cross-sectional area and inertia moment are assumed as $100 \mathrm{~cm}^{2}$ and $833 \mathrm{~cm}^{4}$. The load and vertical-horizontal displacement curves corresponding to the point of imposed load are plotted in Figures 14-15. The relatively higher converged solution is obtained by FEAP. The spherical arc-length method for Opensees is failed due to some difficulties in convergence (see Figures 14b-15b). Although the linearized arc-length method for Opensees is employed with an increase in the number of elements and integral points, it does not show more convergence compared to the linearized arc-length one for FEAP (see 14a and $15 \mathrm{a}$ along with $14 \mathrm{c}$ and $15 \mathrm{c}$ ). However, an increase in the elements number for FEAP leads to obtaining a close equilibrium curve to the relatively higher converged one (see Figure $14 \mathrm{c}$ and $15 \mathrm{c}$ ). The elapsed time for the execution of FEAP is lower than that for Opensees (see Table 2).

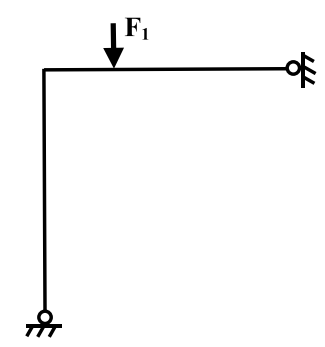

Figure 13. Lee Frame.
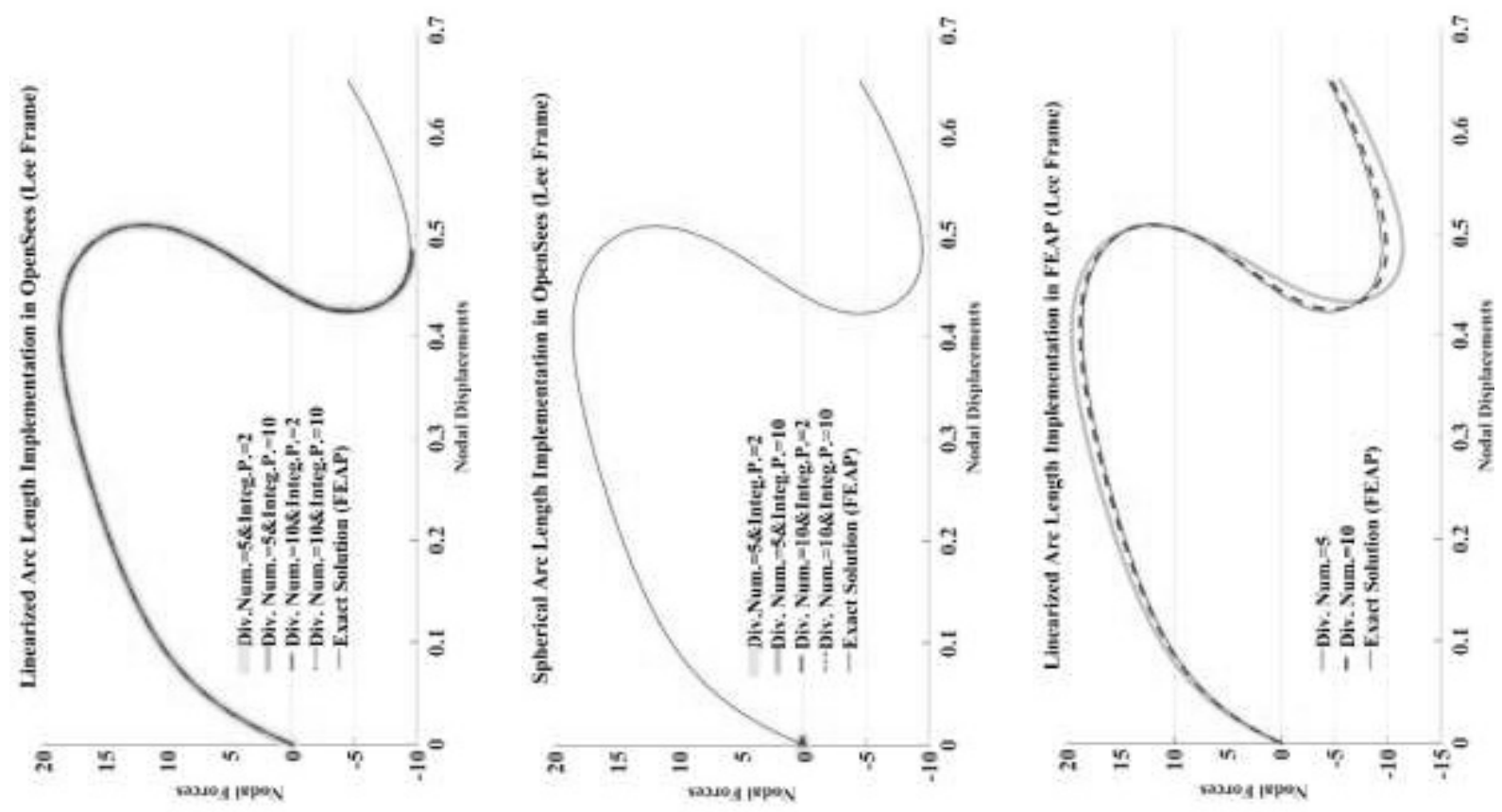

Figure 14. Comparisons of Linearized and Spherical Arc Length Method with an implementation in Opensees along with Spherical Arc Length Method with an implementation in FEAP. 

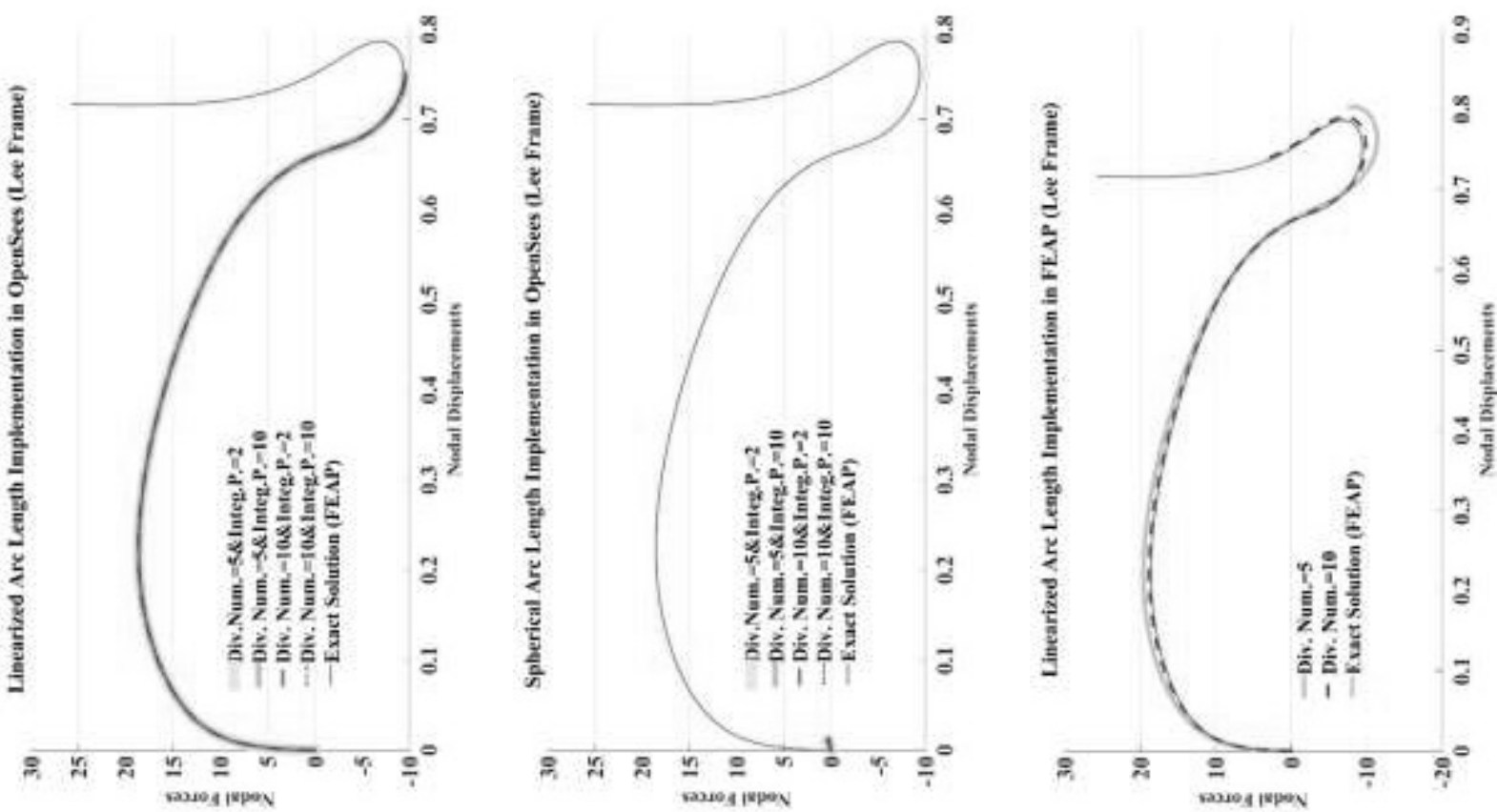

Figure 15. Comparisons of Linearized and Spherical Arc Length Method with an implementation in Opensees along with Spherical Arc Length Method with an implementation in FEAP.

\subsection{Portal Frame Subjected to the Concentrated Loads}

This portal frame shown in Figure 16 has one-bay and one-story. This benchmark problem has been tackled to verify the computer code in working with more practical structures (Hsiao and Huo, 1987). The simple portal frame is subjected to concentrated vertical and horizontal loads of $6000 \mathrm{~N}$ and $6 \mathrm{~N}$ at its upper-left and right-hand nodes. The elasticity module and poison ratio of the portal frame material are taken as $30000 \mathrm{~N} / \mathrm{cm}^{2}$ and 0.3 . The crosssectional area and inertia moment are assumed as $11.77 \mathrm{~cm}^{2}$ and $310.1 \mathrm{~cm}^{4}$. The relatively higher converged solution is obtained by FEAP (see the small figure in Figure 17c). Although the spherical and linearized arc-length methods for Opensees are employed with an increase in the number of elements and integral points, they failed in tracing the full load-displacement curve (see Figure 17a and 17b). However, the increase in the elements number for FEAP leads to showing a good agreement with results in the relatively higher converged solution (see Figure 17c). The elapsed time for the execution of FEAP is lower than that for Opensees (see Table 2).

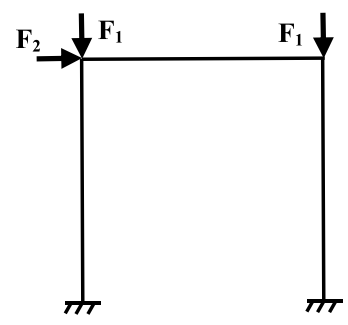

Figure 16. Portal Frame. 

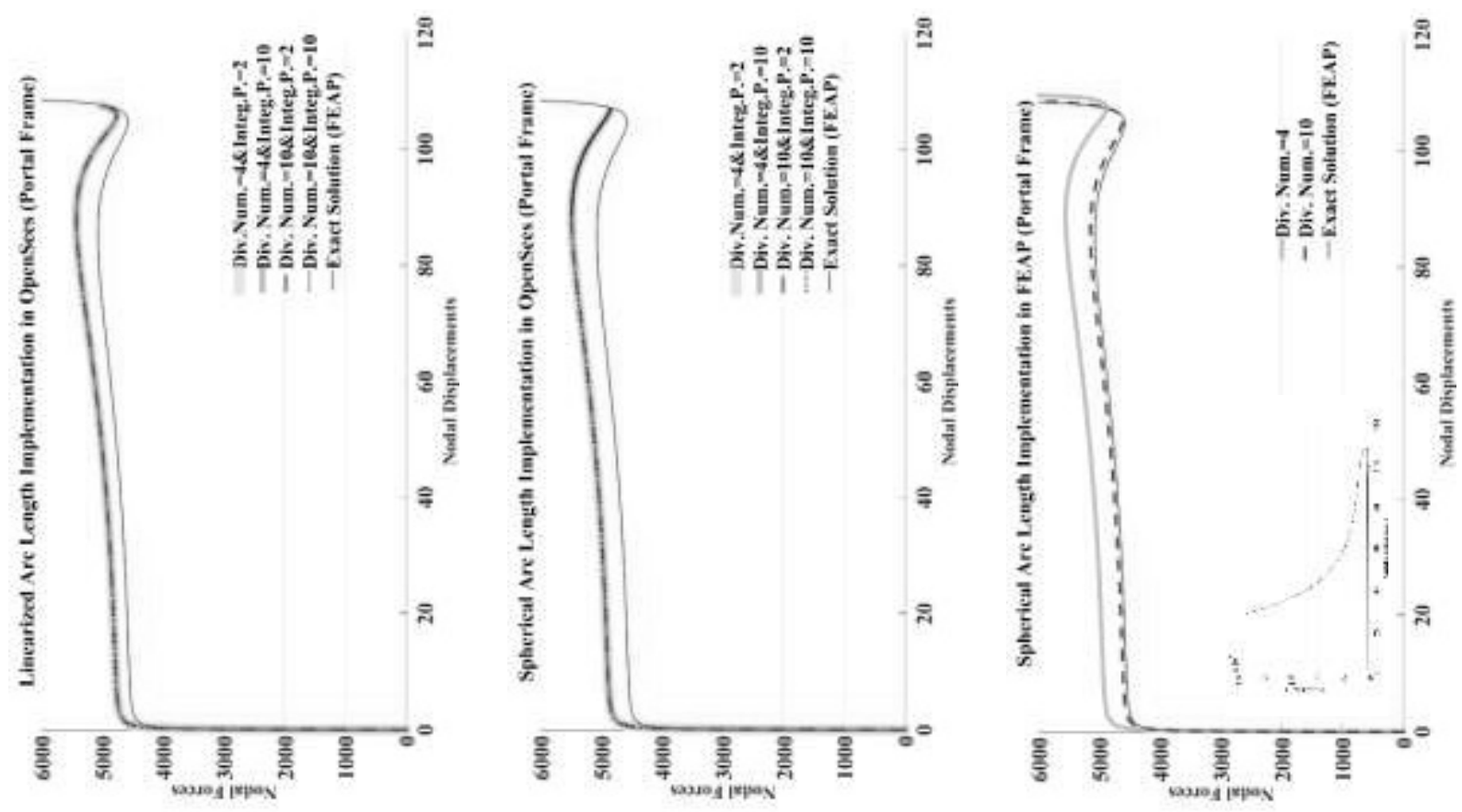

Figure 17. Comparisons of Linearized and Spherical Arc Length Method with an implementation in Opensees along with Spherical Arc Length Method with an implementation in FEAP.

\section{CONCLUSION}

This study proposes to tackle the nonlinearity issue, thereby employing two well-known computer programs with opensource codes, named Opensees and FEAP, for the six benchmark analyses problems. Thus, these computer codes are also evaluated in this study. In this regard, two arc-length methods, which are implemented in the employed computer codes, named spherical and linearized arc length methods, are compared, thereby utilizing two different element formulations. Moreover, in order to increase the convergence degree of the employed solution algorithms, the governing parameters of the element formulation, for example, the numbers of elements and integration points, are combined, assuming their different values, and applied into the benchmark problems. The employed nonlinear solution algorithms are utilized to analyze the six nonlinear benchmark problems. The results obtained are summarized as follows:

i) The computing performance of spherical arc-length technique is the lowest compared to the linearized arc-length technique considering their ability of tracing the equilibrium curve.

ii) The linearized arc-length technique utilized by FEAP exhibits a higher prediction for the equilibrium curve than that for Opensees due to the implementation of a line search approach into its computation mechanism.

iii) Whereas using the increased numbers of elements and integration points for Opensees has not any importance for tracing the equilibrium curve with more convergence degree, FEAP is negatively affected from using a coarse meshing for the discretization of structural system.

iv) The computing time for Opensees is higher than that for FEAP due to the implementation of element state determination in order to establish the stress-strain relation for the flexibility-based element formulation.

\section{ACKNOWLEDGMENT}

The author would like to thank the reviewers for their valuable suggestions and helpful discussions in order to improve the content of this work. Furthermore, it is noted that the author immediately provides the videos of the application examples solved in this work if it is requested. 


\section{REFERENCES}

Carotenuto P. 2006. A three-dimensional finite-deformation small-strain beam model: from a consistent principle of virtual work to the development of a computational finite element approach, University of Pavia, Pavia, Italy.

Cichon, C. 1984. Large displacement in-plane analysis of elastic-plastic frames. Comp. Struct. 19: 737-745.

Chandra, Y., Stanciulescu, I., Eason, T. 2012. Numerical pathologies in snap-through simulations Engineering Structures, 34: 495-504.

Chandra, Y., Zhou, Y., Stanciulescu, I. 2015. A robust composite time integration scheme for snap-through problems Computational Mechanics, 55(5): 1041-1056.

Corsanego, A. \& Tafanelli, A. 1980. Alternative energy techniques for equilibrium stability problems. Meccanica, 15(2): 87-94.

Crisfield, M.A. 1981. A fast incremental/iterative solution procedure that handles snap-through. Comp. Struct. 13(1): 55-62.

Crisfield, M.A. 1990. A consistent co-rotational formulation for non-linear, three-dimensional beam elements. Comp. Methods Appl. Mech. Engrg. 81: 131-150.

Crisfield, M.A. 1997a. Nonlinear Finite Element Analysis of Solid and Struct. Vol I: Essentials, Wiley

Crisfield, M.A. 1997b. Nonlinear Finite Element Analysis of Solid and Struct. Vol 2: Advanced Topics, Wiley

Deppo, D.A.D. \& Schmidt, R. 1975. Instability of clamped-hinged circular arches subjected to a point load. Trans. ASME., 894-896.

Forde, B.W.R. \& Stiemer, S.F. 1987. Improved arc length orthogonality methods for nonlinear finite element analysis. Comp. Struct. 27(5): 625-630.

Fried, I. 1984. Orthogonal trajectory accession to the non-linear equilibrium curve. Comp. Methods in App. Mech. and Engrng. 47: 283-97.

Habibi, A., Bidmeshki, S. 2018. A dual approach to perform geometrically nonlinear analysis of plane truss structures, Steel and Composite Structures, 27(1): 13-25.

Hellinger, E. 1914. Die allgemeine ansätze der mechanik der kontinua. Encyklopädie der Mathematischen Wissenschaften IV. 4: $602-694$.

Hermann L 1965. Elasticity equations for incompressible and nearly incompressible materials by a variational theorem. A.I.A.A. J. 3(10): 1896-1900.

Hsiao, K.M. \& Huo, F.Y. 1987. Nonlinear finite element analysis of elastic frames. Comput. Struct., 26(4): 693-701.

http://projects.ce.berkeley.edu/feap/ (accessed in 2008). FEAP 83

http://opensees.berkeley.edu/ (accessed in 2018). Opensees v2.4

Ibrahimbegovic, A. \& Al-Mikdad, M. 1998. Finite rotations in dynamics of beams and implicit time-stepping schemes. Int. J. Numer. Methods in Eng. 41: 781-814.

Lee, S.L., Manuel, F.S. \& Rossow, E.C. 1968. Large deflection and stability of elastic frames. A.S.C.E. J. Eng. Mech. Div. 94: 521-533.

Marsden, J. 1994. Mathematical foundations of elasticity. Dover publications, Mineola.

Masur, E.F. \& Popelar, C.H. 1976. On the use of the complementary energy in the solution of buckling problems. Int. J. Solids Struct. 12: 203-16.

Moghaddasie, B., Stanciulescu, I. 2013. Direct calculation of critical points in parameter sensitive systems, Computer and Structures, 117: 34-47.

Nanakorn, P. \& Vu, L.N. 2006. A 2D field-consistent beam element for large displacement analysis using the total Lagrangian formulation. Finite Elem. Anal. Des. 42: 1240-1247.

Neuenhofer, A. \& Filippou, F.C. 1997. Evaluation of Nonlinear Frame Finite Element Models. J. of Struct. Engnrg. 123(7): 958-966. 
Nistor, M., Wiebe, R., Stanciulescu, I. 2017. Relationship between Euler buckling and unstable equilibria of buckled beams, International Journal of No-linear Mechanics. 95: 151-161.

Oran, C. 1967. Complementary energy method for buckling. J. Eng. Mech. Div. A.S.C.E. 93: 57-75.

Pacoste, C. \& Eriksson, A. 1997. Beam elements in instability problems. Comput. Method. Appl. Mech. Eng. 144: 163-197.

Planine, I \& Saje, M. 1999. A quadratically convergent algorithm for the computation of stability points: The application of the determinant of the tangent stiffness matrix. Comput. Meth. Appl. Mech. Eng. 169: 89-105.

Ramm, E. 1981. Strategies for tracing the nonlinear response near limit points In Wunderlich, W., Stein, E., \& Bathe, K. J., eds, Nonlinear Finite Element Analysis in Structural Mechanics.

Ray, T. 2016. Enhanced Solution Scheme for Nonlinear Analysis of Force-Based Beam for Large Rotations, Multiple Critical Points, and Random Quasi-Static Loading Input. Journal of Structural Engineering. 142(9): 1943-1968.

Riks, E. 1979. An incremental approach to the solution of snapping and buckling problems. Int. J. Solids Struct. 15(7): 529-551.

Ritto-Correa, M. \& Camotim, D. 2008. On the arc-length and other quadratic control methods: Established, less known and new implementation procedures. Comp. and Struct. 86: 1353-1368.

Ritto-Correa, M. \& Camotim, D. 2002. On the differentiation of the Rodrigues formula and its significance for the vector-like parametrizarion of Reissner-Simo beam theory. International J. for Num. Methods in Eng. 55(9): 1005-1032.

Santos, H. 2011. Complementary-Energy Methods for Geometrically Non-linear Structural Models: An Overview and Recent Developments in the Analysis of Frames Arch. Comp. Methods Eng. 18: 405-440.

Schweizerhof, K. \& Wriggers, P. 1986. Consistent linearization for path following methods in nonlinear fe-analysis. Comp. Methods in App. Mec. and Eng. 59(1): 261-279.

Scott, M.H., Franchin, P., Fenves. G.L. \& Filippou, F.C. 2004. Response Sensitivity for Nonlinear Beam-Column Elements. J. of Struct. Eng. 130(9): 1281-1288.

Scott, M.H. \& Fenves, G.L. 2006. Plastic Hinge Integration Methods for Force-Based Beam-Column Elements. J. of Struct. Eng., 132(2): 244-252.

Scott, M.H., Fenves, G.L., McKenna, F.T., \& Filippou, F.C. 2008. Software Patterns for Nonlinear Beam-Column Models. J. of Struct. Eng. 134(4): 562-568.

Shames, I.H. \& Dym, C.L. 1985. Energy and finite element methods in structural mechanics. McGraw-Hill, New York.

Shokrieh, M.M., Parkestani, A., Nonni 2017. Post buckling analysis of shallow composite shells based on the third order shear deformation theory. Aerospace Science and Tchnology. 66: 332-341.

Simo, J.C. \& Vu-Quoc, L. 1986. A three-dimensional finite-strain rod model, Part II: Computational aspects. Comput. Methods Appl. Mech. Eng. 58: 79-116.

Smolenski, W.M. 1999. Statically and kinematically exact nonlinear theory of rods and its numerical verification. Comput. Meth. Appl. Mech. Eng. 178: 89-113.

Souza, R.M. 2000. Force-based Finite Element for Large Displacement Inelastic Analysis of Frames, University of California, Berkeley, U.S.A.

Spacone, E., Ciampi, V., \& Filippou, F.C. 1996. Mixed Formulation of Nonlinear Beam Finite Element. Comp. and Struct., 58(1): 71-83.

Tabarrok, B. \& Yuexi, X. 1989. Application of a new variational formulation for stability analysis of columns subjected to distributed loads. Z.A.M.M. 69(12): 435-40.

Teng, J.G. \& Luo, Y.F. 1998. A user-controlled arc-length method for convergence to prede-fined deformation states. Commun. in Num. Methods in Eng. 14: 51-58.

Uddin, M., Sheikh, A.H., Brown, D. 2018. Geometrically nonlinear inelastic analysis of steel-concrete composite beams with partial interaction using a higher-order beam theory, International Journal of Nonlinear Mech. 100: 34-47.

Veubeke, B. 1965. Stress analysis. Wiley, New York. 
Williams, F.M. 1964. An approach to the nonlinear behavior of members of a rigid jointed plane framework with finite deflections. Quarter Applied Mathematics, 17: 451-469.

Wood, R.D. \& Zienkiewicz, O.C. 1977. Geometrically nonlinear finite element analysis of beams, frames, arches and axisymmetric shells. Comp. and Struct. 7: 725-735.

Xiao-Zu, S. \& Bashir-Ahmed, M. 2004. Arc-length technique for nonlinear finite element analysis. Journal of Zhejiang University SCIENCE. 5(5): 618-628.

Yuan Z. \& Kardomateas G.A. 2018. Nonlinear Stability Analysis of Sandwich Wide Panels—Part II: Postbuckling Response. ASME. J. Appl. Mech. 85(8): 081007-081007-9. doi:10.1115/1.4039954.

Zienkiewicz, O.C. \& Taylor, R.L. 2000a. The Finite Element Method. Volume 1: The Basis. Butterworth-Heinemann, Oxford.

Zienkiewicz, O.C. \& Taylor, R.L. 2000b. The Finite Element Method. Volume 2: Solid Mechanic. Butterworth-Heinemann, Oxford. 